\title{
GRADIENT ESTIMATES FOR PARABOLIC AND ELLIPTIC SYSTEMS FROM LINEAR LAMINATES
}

\author{
HONGJIE DONG
}

\begin{abstract}
We establish several gradient estimates for second-order divergence type parabolic and elliptic systems. The coefficients and data are assumed to be Hölder or Dini continuous in the time variable and all but one spatial variables. This type of systems arises from the problems of linearly elastic laminates and composite materials. For the proof, we use Campanato's approach in a novel way. Non-divergence type equations under a similar condition are also discussed.
\end{abstract}

\section{Contents}

1. Introduction

2. Notation and main results 4

3. Some auxiliary estimates 9

4. Systems with partially Dini coefficients

5. Systems with partially Hölder coefficients 18

6. Non-divergence form equations 23

7. Appendix 29

Acknowledgement $\quad 30$

References $\quad 30$

\section{IntRoduction}

We consider second-order divergence type parabolic and elliptic systems with coefficients and data which are irregular in one of spatial directions. This type of systems arises from the problems of linearly elastic laminates and composite materials. We are interested in the local regularity of the gradient of weak solutions to these systems.

Problems of this kind have been studied by many authors; see, for instance, [3, 2, 1, 13, 14]. In [3], Chipot, Kinderlehrer, and Vergara-Caffarelli considered the weak variational formulation of the equilibrium problem of a linear laminates, i.e. a domain in $\mathbb{R}^{d}$ consisting of a finite number $M$ of

2010 Mathematics Subject Classification. 35R05,35J55.

Key words and phrases. Second-order systems, partially Hölder coefficients, partially Dini coefficients.

H. Dong was partially supported by the NSF under agreements DMS-0800129 and DMS-1056737. 
linearly elastic, homogeneous, parallel laminae. They proved that any weak solution $u$ of the uniformly elliptic system $\operatorname{div}(A \nabla u)=f$ is actually locally Lipschitz, under the conditions that $f$ is in $H^{k}(\Omega), k \geq[d / 2]$, and the coefficients matrix $A$ are constants in each parallel laminae. They also showed that the $W^{1, \infty}$ norm of $u$ is independent of the number $M$, so that in the limiting case $A$ are allowed to be functions of one direction alone. In [13], $\mathrm{Li}$ and Vogelius considered scalar elliptic equations for a single real function $u$ :

$$
\operatorname{div}(A \nabla u)=f+\operatorname{div} g
$$

in a domain which consists a finite number $M$ of disjoint sub-domains with $C^{1, \alpha}$ boundaries. This equation models deformations in composite media such as fiber-reinforced materials. The matrix $A$ and data are assumed to be Hölder continuous up to the boundary in each sub-domains, but may have jump discontinuities across the boundaries of the sub-domains. Under these assumptions, the authors derived global $W^{1, \infty}$ and piecewise $C^{1, \delta}$ estimates of the solution $u$ for $\delta \in\left(0, \frac{\alpha}{d(\alpha+1)}\right]$. Their results were later extended to elliptic systems for vector-valued function $u$ by Li and Nirenberg 14 under the same conditions, and the range of $\delta$ was also relaxed to $\left(0, \frac{\alpha}{2(\alpha+1)}\right]$. The bounds obtained in [13, 14, however, may depend on the number $M$. The corresponding problem for parabolic systems is more complicated. In a forthcoming paper, $\mathrm{Li}$ and $\mathrm{Li}$ [15] further extend some results in [14] to parabolic systems under an additional assumption that the coefficients and data are at least twice differentiable in $t$. See also [7] for a related result on parabolic systems.

We would like to mention two recent papers [5] and [19] on "partial Schauder" estimates. In [5], Dong and Kim considered both divergence and non-divergence form second-order scalar elliptic and parabolic equations. They proved that if the coefficients and data are Hölder continuous in some directions, then derivatives of solutions in these directions are Hölder continuous in the same directions. By using a different method, Tian and Wang [19] proved similar results for non-divergence form elliptic equations with coefficients and data Dini continuous in some variables. Under certain conditions, their results also extend to second-order fully nonlinear equations. An interesting question is how much regularity one can expect in the "bad" directions. In this paper we address this question when the "bad" direction is one-dimensional. We note that the proofs in [5] and [19] do not apply to systems since the maximum principle is used in both papers. In the case that the coefficients are regular in all directions, a similar problem was studied long time ago by Fife [8].

In this paper, we are concerned with parabolic and elliptic systems:

$$
\begin{aligned}
\mathcal{P} u & :=-u_{t}+D_{\alpha}\left(A^{\alpha \beta} D_{\beta} u\right)+D_{\alpha}\left(B^{\alpha} u\right)+\hat{B}^{\alpha} D_{\alpha} u+C u=\operatorname{div} g+f, \\
\mathcal{L} u & :=D_{\alpha}\left(A^{\alpha \beta} D_{\beta} u\right)+D_{\alpha}\left(B^{\alpha} u\right)+\hat{B}^{\alpha} D_{\alpha} u+C u=\operatorname{div} g+f .
\end{aligned}
$$


The coefficients of $\mathcal{P}$ and $\mathcal{L}$ are assumed to be bounded, and the operators are uniformly nondegenerate. The aim of our paper is to obtain optimal regularity of weak solutions when coefficients are assumed to be regular in the time variable and all but one spatial variables. To be more precise, let us denote a typical point in $\mathbb{R}^{d+1}$ by $z=(t, x)$, where $x=\left(x^{1}, \cdots, x^{d}\right):=$ $\left(x^{\prime}, x^{d}\right)$ and $z^{\prime}:=\left(t, x^{\prime}\right)$. We shall prove that if the coefficients and data are Dini continuous in $z^{\prime}$, then any weak solution $u$ to (1.1) is locally Lipschitz in all spatial variables, $C^{1 / 2}$ in $t$, and $D_{x^{\prime}} u$ and $\hat{U}:=A^{d \beta} D_{\beta} u+B^{d} u-g_{d}$ are continuous; see Theorem 2.1 below for more precise statement. We also prove that a Hölder regularity assumption in $z^{\prime}$ on the coefficients and data gives a better regularity of $u$. In particular, $D_{x^{\prime}} u$ and $\hat{U}$ are Hölder in all variables; see Theorem 2.2 .

In the special case that the domain consists of $M$ parallel laminate subdomains as in [3] mentioned above, we show that if the coefficients and data are regular in each sub-domain and may have jump discontinuous across the boundaries, then $D u$ is regular in each sub-domain up to the boundary; see Remark 2.5 i). Thus our results generalize the aforementioned results in 3 ] by allowing more general coefficients and also deriving optimal $C^{1, \delta}$ estimate for $\delta \in(0,1)$. Unlike [13, 14], we do not impose any restriction on $\delta$, and the bounds of various norms are independent of $M$. However, it should be pointed out that although we allow subdomains to have curved boundaries (see Remark 2.5 ii)), the geometry of the domain considered in [13, 14] is more general than in the current paper.

Our arguments are quite different from those in [13, 14, 15] and [5, 19]. Let us give a brief description as follows. The proofs below are based on Campanato's approach, which was used previously, for instance, in [9, 16]. The main step of Campanato's approach is to show the mean oscillations of $D u$ in balls vanish in certain order as the radii of balls go to zero. However, we are not able to follow this approach in the usual way due to the lack of regularity of $u$ in the $x^{d}$-direction. To overcome this difficulty, we appeal to a recent result in [4] regarding the $L_{p}$ estimate for systems with partially VMO coefficients. A crucial step in the proof is to deduce from this result some interior Hölder regularity of $D_{x^{\prime}} u$ and $U:=A^{d \beta} D_{\beta} u$ for parabolic systems with coefficients depending on $x^{d}$ alone. We then use some perturbation arguments on $D_{x^{\prime}} u$ and $U$ together with a certain decomposition of $u$ to get the desired estimates.

By using a similar idea, we also obtain the corresponding results for scalar non-divergence form parabolic equations:

$$
P u:=-u_{t}+a^{\alpha \beta} D_{\alpha \beta} u+b^{\alpha} D_{\alpha} u+c u=f .
$$

We prove that if the coefficients and $f$ are Dini continuous in $z^{\prime}$, then any solution $u$ of the above equation is $C^{1}$ in $t, C^{1,1}$ in $x$, and $u_{t}$ and $D_{x x^{\prime}} u$ are continuous; see Theorem 6.1. Under the stronger condition that the coefficients and $f$ are Hölder continuous in $z^{\prime}$, we obtain additionally that $u_{t}$ and $D_{x x^{\prime}} u$ are Hölder continuous in all variables; see Theorem 6.2. These 
theorems generalize some results in [8, 5, 19] for the Poisson equation. In the case that the domain consists of $M$ parallel laminate sub-domains and the coefficients and $f$ are regular in each sub-domain, we show that the second derivative of $u$ in the $x^{d}$-direction is also Hölder continuous up to the boundary in each sub-domain.

As mentioned above, some of our estimates rely on recent work about $L_{p}$-regularity for elliptic and parabolic equations (systems) with leading coefficients VMO in some of the independent variables; see Section 3. This series of work was initiated by Krylov in 12. For further developments in this direction, we also refer the reader to [10, 4, 6] and references therein.

The organization of this paper is as follows. In Section 2, we state our main theorems for divergence form systems and introduce some notation. We prove some auxiliary estimates in Section 3. The proofs of main theorems are given in Section 4 and Section 5. Finally we treat non-divergence form scalar equations in Section 6 .

\section{Notation And MAin RESUlts}

We are concerned with parabolic systems

$$
\mathcal{P} u:=-u_{t}+D_{\alpha}\left(A^{\alpha \beta} D_{\beta} u\right)+D_{\alpha}\left(B^{\alpha} u\right)+\hat{B}^{\alpha} D_{\alpha} u+C u=\operatorname{div} g+f,
$$

where $g=\left(g_{1}, g_{2}, \cdots, g_{d}\right)$. The coefficients $A^{\alpha \beta}, B^{\alpha}, \hat{B}^{\alpha}, C$ are $n \times n$ matrices, which are bounded by a positive constant $K$, and the leading coefficient matrices $A^{\alpha \beta}$ are uniformly elliptic with ellipticity constant $\nu$ :

$$
\nu|\xi|^{2} \leq A_{i j}^{\alpha \beta} \xi_{i}^{\alpha} \xi_{j}^{\beta}, \quad\left|A^{\alpha \beta}\right| \leq \nu^{-1}
$$

for any $\xi=\left(\xi_{i}^{\alpha}\right) \in \mathbb{R}^{d \times n}$. Here

$$
u=\left(u^{1}, \cdots, u^{n}\right)^{\operatorname{tr}}, \quad g_{\alpha}=\left(g_{\alpha}^{1}, \cdots, g_{\alpha}^{n}\right)^{\operatorname{tr}}, \quad f=\left(f^{1}, \cdots, f^{n}\right)^{\operatorname{tr}}
$$

are (column) vector-valued functions. Throughout the paper, the summation convention over repeated indices is used. We also consider the following elliptic system

$$
\mathcal{L} u:=D_{\alpha}\left(A^{\alpha \beta} D_{\beta} u\right)+D_{\alpha}\left(B^{\alpha} u\right)+\hat{B}^{\alpha} D_{\alpha} u+C u=\operatorname{div} g+f .
$$

In this case $A^{\alpha \beta}, B^{\alpha}, \hat{B}^{\alpha}, C, g$, and $f$ are independent of $t$ and satisfy the same conditions as in the parabolic case.

2.1. Notation. By $D u=\left(D_{i} u\right)$ and $D^{2} u=\left(D_{i j} u\right)$ we mean the gradient and the Hessian matrix of $u$. On many occasions we need to take these objects relative to only part of variables. We also use the following notation:

$$
D_{t} u=u_{t}, \quad D_{x^{\prime}} u=u_{x^{\prime}}, \quad D_{x x^{\prime}} u=u_{x x^{\prime}} .
$$

Set

$$
\begin{gathered}
B_{r}^{\prime}\left(x^{\prime}\right)=\left\{y \in \mathbb{R}^{d-1}:\left|x^{\prime}-y^{\prime}\right|<r\right\}, \quad B_{r}(x)=\left\{y \in \mathbb{R}^{d}:|x-y|<r\right\}, \\
Q_{r}^{\prime}(t, x)=\left(t-r^{2}, t\right) \times B_{r}^{\prime}\left(x^{\prime}\right), \quad Q_{r}(t, x)=\left(t-r^{2}, t\right) \times B_{r}(x),
\end{gathered}
$$


and

$$
B_{r}^{\prime}=B_{r}^{\prime}(0), \quad B_{r}=B_{r}(0), \quad Q_{r}^{\prime}=Q_{r}^{\prime}(0,0), \quad Q_{r}=Q_{r}(0,0) .
$$

By $N(d, p, \cdots)$ we mean that $N$ is a constant depending only on the prescribed quantities $d, p, \cdots$. For a (matrix-valued) function $f(t, x)$ in $\mathbb{R}^{d+1}$, we set

$$
(f)_{\mathcal{D}}=\frac{1}{|\mathcal{D}|} \int_{\mathcal{D}} f(t, x) d x d t=f_{\mathcal{D}} f(t, x) d x d t,
$$

where $\mathcal{D}$ is an open subset in $\mathbb{R}^{d+1}$ and $|\mathcal{D}|$ is the $d+1$-dimensional Lebesgue measure of $\mathcal{D}$.

2.2. Lebesgue spaces. For $p \in(1, \infty)$, we denote

$$
W_{p}^{1,2}(\mathcal{D})=\left\{u: u, u_{t}, D u, D^{2} u \in L_{p}(\mathcal{D})\right\} .
$$

We also denote $\mathbb{H}_{p}^{-1}(\mathcal{D})$ to be the space consisting of all functions $u$ satisfying

$$
\inf \left\{\|g\|_{L_{p}(\mathcal{D})}+\|h\|_{L_{p}(\mathcal{D})} \mid u=\operatorname{div} g+h\right\}<\infty .
$$

It is easy to see that $\mathbb{H}_{p}^{-1}(\mathcal{D})$ is a Banach space. Naturally, for any $u \in$ $\mathbb{H}_{p}^{-1}(\mathcal{D})$, we define the norm

$$
\|u\|_{\mathbb{H}_{p}^{-1}(\mathcal{D})}=\inf \left\{\|g\|_{L_{p}(\mathcal{D})}+\|h\|_{L_{p}(\mathcal{D})} \mid u=\operatorname{div} g+h\right\} \text {. }
$$

We also define

$$
\mathcal{H}_{p}^{1}(\mathcal{D})=\left\{u: u, D u \in L_{p}(\mathcal{D}), u_{t} \in \mathbb{H}_{p}^{-1}(\mathcal{D})\right\} .
$$

We use the abbreviations $W_{p}^{1,2}=W_{p}^{1,2}\left(\mathbb{R}^{d+1}\right)$ and $\mathcal{H}_{p}^{1}=\mathcal{H}_{p}^{1}\left(\mathbb{R}^{d+1}\right)$, etc.

2.3. Partially VMO and partially Dini spaces. For a function $u$ in $\mathcal{D}$, we define its modulus of continuity $\omega_{u, z^{\prime}}$ (in the mean) with respect to $z^{\prime}$ by

$$
\begin{aligned}
& \omega_{u, z^{\prime}}(R) \\
& =\sup _{\substack{z_{0} \in \mathbb{R}^{d+1} \\
r \leq R}}\left(\left|Q_{r}\right|^{-2} \int_{Q_{r}\left(z_{0}\right) \cap \mathcal{D}} \int_{Q_{r}\left(z_{0}\right) \cap \mathcal{D}}\left|u\left(t, x^{\prime}, x^{d}\right)-u\left(s, y^{\prime}, x^{d}\right)\right|^{2} d y d s d x d t\right)^{\frac{1}{2}} .
\end{aligned}
$$

We say $u$ is partially VMO with respect to $z^{\prime}$ if $\omega_{u, z^{\prime}}(R) \rightarrow 0$ as $R \rightarrow 0$.

We call a continuous increasing function $\omega$ on $\overline{\mathbb{R}^{+}}$a Dini function if $\omega(0)=$ 0 and for any $t>0$

$$
I[\omega](t):=\int_{0}^{t} \omega(s) / s d s<\infty .
$$

We say function $u$ in $\mathcal{D}$ is partially Dini continuous with respect to $z^{\prime}$ if its modulus of continuity $\omega_{u, z^{\prime}}$ in $z^{\prime}$ is a Dini function. In this case, we write $u \in C_{z^{\prime}}^{\text {Dini }}(\mathcal{D})$. Clearly, any function in $C_{z^{\prime}}^{\text {Dini }}(\mathcal{D})$ is partially VMO with respect to $z^{\prime}$.

In a similar way, in the time independent case we define $\omega_{u, x^{\prime}}$ and the space $C_{x^{\prime}}^{\text {Dini }}$. 
We note that our definition of Dini continuity is slightly different from the usual definition, where the modulus of continuity is measured in the uniform sense.

2.4. Hölder spaces. For $\delta \in(0,1]$, we denote the $C^{\delta / 2, \delta}$ semi-norm by

$$
[u]_{\delta / 2, \delta ; \mathcal{D}}:=\sup _{\substack{(t, x),(s, y) \in \mathcal{D} \\(t, x) \neq(s, y)}} \frac{|u(t, x)-u(s, y)|}{|t-s|^{\delta / 2}+|x-y|^{\delta}}
$$

and the $C^{\delta / 2, \delta}$ norm by

$$
|u|_{\delta / 2, \delta ; \mathcal{D}}:=[u]_{\delta / 2, \delta ; \mathcal{D}}+|u|_{0 ; \mathcal{D}}
$$

where $|u|_{0 ; \mathcal{D}}=\sup _{\mathcal{D}}|u|$. Next we define

$$
\begin{aligned}
{[u]_{(1+\delta) / 2,1+\delta ; \mathcal{D}}:=[D u]_{\delta / 2, \delta ; \mathcal{D}}+\langle u\rangle_{1+\delta ; \mathcal{D}}, } \\
|u|_{(1+\delta) / 2,1+\delta ; \mathcal{D}}:=[u]_{(1+\delta) / 2,1+\delta ; \mathcal{D}}+|u|_{0 ; \mathcal{D}}+|D u|_{0 ; \mathcal{D}},
\end{aligned}
$$

where

$$
\langle u\rangle_{1+\delta ; \mathcal{D}}:=\sup _{\substack{(t, x),(s, x) \in \mathcal{D} \\ t \neq s}} \frac{|u(t, x)-u(s, x)|}{|t-s|^{(1+\delta) / 2}} .
$$

By $C^{(1+\delta) / 2,1+\delta}(\mathcal{D})$ we denote the set of all bounded measurable functions $u$ for which the derivatives $D u$ are continuous and bounded in $\mathcal{D}$ and $[u]_{(1+\delta) / 2,1+\delta ; \mathcal{D}}<\infty$.

We define a partial Hölder semi-norm with respect to $z^{\prime}$ as

$$
[u]_{z^{\prime}, \delta / 2, \delta ; \mathcal{D}}:=\sup _{\substack{(t, x),(s, y) \in \mathcal{D} \\ x^{d}=y^{d},(t, x) \neq(s, y)}} \frac{|u(t, x)-u(s, y)|}{|t-s|^{\delta / 2}+|x-y|^{\delta}},
$$

and the corresponding norm as

$$
|u|_{z^{\prime}, \delta / 2, \delta ; \mathcal{D}}:=[u]_{z^{\prime}, \delta / 2, \delta ; \mathcal{D}}+|u|_{0 ; \mathcal{D}} .
$$

By $C_{z^{\prime}}^{\delta / 2, \delta}(\mathcal{D})$ we denote the set of all bounded measurable functions $u$ on $\mathcal{D}$ for which $[u]_{z^{\prime}, \delta / 2, \delta ; \mathcal{D}}<\infty$. Similarly, we define

$$
[u]_{z^{\prime},(1+\delta) / 2,1+\delta ; \mathcal{D}}:=\left[D_{x^{\prime}} u\right]_{z^{\prime}, \delta / 2, \delta ; \mathcal{D}}+\langle u\rangle_{1+\delta ; \mathcal{D}},
$$

and the space $C_{z^{\prime}}^{(1+\delta) / 2,1+\delta}(\mathcal{D})$. In the time-independent case, we also define $[\cdot]_{x^{\prime}, \delta},|\cdot|_{x^{\prime}, \delta}$ and the space $C_{x^{\prime}}^{\delta}$ in a similar fashion.

2.5. Main results. We state the main results of the paper concerning divergence form parabolic systems. Roughly speaking, the first theorem reads if the coefficients and data are Dini continuous in $z^{\prime}$, then any weak solution $u$ to (2.1) is Lipschitz in all spatial variables and 1/2-Hölder in $t$. 
Theorem 2.1. Let $A \in C_{z^{\prime}}^{D i n i}, B \in C_{z^{\prime}}^{D i n i}, f \in L_{\infty}\left(Q_{1}\right)$ and $g \in C_{z^{\prime}}^{D i n i}\left(Q_{1}\right)$. Assume that $u$ is a weak solution to (2.1) in $Q_{1}$. Then we have $u \in$ $C^{1 / 2,1}\left(Q_{1 / 2}\right)$ and

$$
|u|_{1 / 2,1 ; Q_{1 / 2}} \leq N\left(I\left[\omega_{g, z^{\prime}}\right](1)+|g|_{0 ; Q_{1}}+|f|_{0 ; Q_{1}}+\|u\|_{L_{2}\left(Q_{1}\right)}\right),
$$

where $N=N\left(d, n, \nu, K, \omega_{A, z^{\prime}}, \omega_{B, z^{\prime}}\right)$. Moreover, $D_{x^{\prime}} u$ and $\hat{U}$ are continuous in $\overline{Q_{1 / 2}}$, where

$$
\hat{U}:=A^{d \beta} D_{\beta} u+B^{d} u-g_{d} .
$$

In the next theorem, we show that a partial Hölder regularity assumption on the coefficients and data gives a better regularity of $u$. In particular, the spatial derivatives of $u$ in $x^{\prime}$ and $\hat{U}$ are Hölder continuous in all variables.

Theorem 2.2. Let $\delta \in(0,1), A \in C_{z^{\prime}}^{\delta / 2, \delta}, B \in C_{z^{\prime}}^{\delta / 2, \delta}, f \in L_{\infty}\left(Q_{1}\right)$ and $g \in C_{z^{\prime}}^{\delta / 2, \delta}\left(Q_{1}\right)$. Assume that $u$ is a weak solution to (2.1) in $Q_{1}$. Then we have $u \in C^{1 / 2,1}\left(Q_{1 / 2}\right), D_{x^{\prime}} u, \hat{U} \in C^{\delta / 2, \delta}\left(Q_{1 / 2}\right)$ and

$$
\begin{aligned}
|D u|_{0 ; Q_{1 / 2}}+\langle u\rangle_{1+\delta ; Q_{1 / 2}}+ & {\left[D_{x^{\prime}} u\right]_{\delta / 2, \delta ; Q_{1 / 2}}+[\hat{U}]_{\delta / 2, \delta ; Q_{1 / 2}} } \\
& \leq N\left(|g|_{z^{\prime}, \delta / 2, \delta ; Q_{1}}+|f|_{0 ; Q_{1}}+\|u\|_{L_{2}\left(Q_{1}\right)}\right)
\end{aligned}
$$

where $N=N\left(d, n, \delta, \nu, K,[A]_{z^{\prime}, \delta / 2, \delta},[B]_{z^{\prime}, \delta / 2, \delta}\right)$.

Remark 2.3. It follows from the definition of $\hat{U}$ that $D_{d} u$ is continuous in $z^{\prime}$ in Theorem 2.1, and is in $C_{z^{\prime}}^{\delta / 2, \delta}$ in Theorem 2.2. In other words, $D_{d} u$ is only discontinuous in the $x^{d}$ direction, which is a quite intuitive phenomenon from a physical point of view.

Remark 2.4. The conditions on $f, g$ and $B$ in Theorems 2.1 and 2.2 can be relaxed. From the proofs below, it is easily seen that we only need $f$ to be in some weaker Morrey space (cf. (4.3)). For $g_{\alpha}, B^{\alpha}, \alpha=1, \cdots, d-1$, we only require the regularity with respect to the $x^{\alpha}$-direction, in which the derivative is taken.

Remark 2.5. i) Suppose that $Q_{1}$ is divided into $M$ laminate sub-domains $\mathcal{D}_{1}, \mathcal{D}_{2}, \cdots, \mathcal{D}_{M}$ by $M-1$ parallel hyperplanes with the common normal direction $(0, \cdots, 0,1)$. Under the conditions of Theorem 2.2 , we assume in addition that $A^{d \beta}, \beta=1, \cdots, d, B^{d}$ and $g_{d}$ are in $C^{\delta / 2, \delta}\left(\overline{\mathcal{D}_{i}}\right)$ for each $i=1,2, \cdots, M$, but may have jump discontinuities across these hyperplanes. Then we infer that

$$
D_{d} u=\left(A^{d d}\right)^{-1}\left(\hat{U}+g_{d}-B^{d} u-\sum_{\beta=1}^{d-1} A^{d \beta} D_{\beta} u\right)
$$

is also in $C^{\delta / 2, \delta}\left(\overline{\mathcal{D}_{i}} \cap Q_{1-\varepsilon}\right)$ for any $i=1, \cdots, M$ and $\varepsilon \in(0,1 / 2)$, with the $C^{\delta / 2, \delta}$ norms independent of $M$. Similarly, in Theorem 2.1, if we additionally assume that $A^{d \beta}, \beta=1, \cdots, d, B^{d}$ and $g_{d}$ are piecewise continuous, then $D_{d} u$ is also continuous in $\overline{\mathcal{D}_{i}} \cap Q_{1-\varepsilon}$. 
ii) Our results can be extended to systems which model composite materials similar to those considered in [13, 14]. Let $T>0$, and $\Omega$ be a bounded domain in $\mathbb{R}^{d}$ which consists $M$ disjoint subdomains $\Omega_{1}, \cdots, \Omega_{M}$ with $C^{1, \delta}$ boundaries. Suppose that any point belongs to the boundaries of at most two of the subdomains. We also assume that $A^{\alpha \beta}, B^{\alpha}$ and $g_{\alpha}$ are in $C^{\delta / 2, \delta}\left((-T, 0] \times \overline{\Omega_{i}}\right)$ for each $i=1,2, \cdots, M$. For $\varepsilon>0$, denote

$$
\Omega_{\varepsilon}=\{x \in \Omega: \operatorname{dist}(x, \partial \Omega)>\varepsilon\} .
$$

Let $u$ be a weak solution to (2.1) in $(-T, 0) \times \Omega$. Then by the standard technique of locally flattening the boundaries, we can apply Theorem 2.2 to obtain that

$$
u \in C^{(1+\delta) / 2,1+\delta}\left((-T+\varepsilon, 0] \times\left(\Omega_{\varepsilon} \cap \overline{\Omega_{i}}\right)\right)
$$

for any $i=1, \cdots, M$ and $\varepsilon \in(0,1 / 2)$. Similarly, if $\Omega_{1}, \cdots, \Omega_{M}$ have $C^{1 \text {,Dini }}$ boundaries and $A^{\alpha \beta}, B^{\alpha}, g_{\alpha}$ are Dini continuous in $(-T, 0] \times \overline{\Omega_{i}}$ for each $i=1,2, \cdots, M$, then from Theorem 2.1 we get

$$
u \in C^{1 / 2,1}\left((-T+\varepsilon, 0] \times\left(\Omega_{\varepsilon} \cap \overline{\Omega_{i}}\right)\right), \quad i=1, \cdots, M,
$$

and $D u$ is continuous in $(-T+\varepsilon, 0] \times\left(\Omega_{\varepsilon} \cap \overline{\Omega_{i}}\right)$. However, the bounds of $u$ may also depends on the distances between different inclusions. Therefore, this method does not apply to the case when the boundaries of three subdomains touch at some point. We remark that the estimates obtained in [13, 14 (see also recently [15] and [7]) are independent of the distances between inclusions, under a restriction on the range of $\delta$. Estimates of this type are interesting from a physical point of view because, in the elliptic model of extreme valued conductivity, the gradient blows up as the distance goes to zero.

Next we state the results for elliptic systems (2.2), which follow immediately from Theorems 2.1 and 2.2 by viewing the solutions to the elliptic systems as a steady state solution to the corresponding parabolic systems.

Corollary 2.6. Let $A \in C_{x^{\prime}}^{\text {Dini }}, B \in C_{x^{\prime}}^{\text {Dini }}, f \in L_{\infty}\left(B_{1}\right)$ and $g \in C_{x^{\prime}}^{\text {Dini }}\left(B_{1}\right)$. Assume that $u$ is a weak solution to (2.2) in $B_{1}$. Then we have

$$
|D u|_{0 ; B_{1 / 2}} \leq N\left(I\left[\omega_{g, z^{\prime}}\right](1)+|g|_{0 ; B_{1}}+|f|_{0 ; B_{1}}+\|u\|_{L_{2}\left(B_{1}\right)}\right),
$$

where $N=N\left(d, n, \nu, K, \omega_{A, z^{\prime}}, \omega_{B, z^{\prime}}\right)$. Moreover, $D_{x^{\prime}} u$ and $\hat{U}$ are continuous in $\overline{B_{1 / 2}}$.

Corollary 2.7. Let $\delta \in(0,1), A \in C_{x^{\prime}}^{\delta}, B \in C_{x^{\prime}}^{\delta}, f \in L_{\infty}\left(B_{1}\right)$ and $g \in$ $C_{x^{\prime}}^{\delta}\left(B_{1}\right)$. Assume that $u$ is a weak solution to $(2.2)$ in $B_{1}$. Then we have

$$
|D u|_{0 ; B_{1 / 2}}+\left[D_{x^{\prime}} u\right]_{\delta ; B_{1 / 2}}+[\hat{U}]_{\delta ; B_{1 / 2}} \leq N\left(|g|_{x^{\prime}, \delta ; B_{1}}+|f|_{0 ; B_{1}}+\|u\|_{L_{2}\left(B_{1}\right)}\right),
$$

where $N=N\left(d, n, \delta, \nu, K,[A]_{x^{\prime}, \delta},[B]_{x^{\prime}, \delta}\right)$.

Estimates in the same spirit as in Theorems 2.1 and2.2 for non-divergence form equations are stated in Section 6 . 


\section{Some AUXiliary estimates}

We will use the following property of Dini functions.

Lemma 3.1. Suppose that $\omega$ is a Dini function, and

$$
\tilde{\omega}(t):=\sum_{k=0}^{\infty} a^{k}\left(\omega\left(b^{k} t\right) \chi_{b^{k} t \leq 1}+\omega(1) \chi_{b^{k} t>1}\right)
$$

for some constants $a \in(0,1)$ and $b>1$. Then $\tilde{\omega}$ is also a Dini function.

Proof. Let $\hat{\omega}(t)=\omega(t)$ for $t \leq 1$ and $\hat{\omega}(t)=\omega(1)$ for $t>1$. Then $\hat{\omega}$ is bounded, uniformly continuous Dini function. Moreover,

$$
\tilde{\omega}(t)=\sum_{k=0}^{\infty} a^{k} \hat{\omega}\left(b^{k} t\right) .
$$

Then it is easy to see that $\tilde{\omega}$ is increasing and continuous. It follows from (3.1) that

$$
\tilde{\omega}(t) \leq \sum_{k=0}^{\infty} a^{k} \omega\left(b^{k} t\right) \chi_{b^{k} t \leq 1}+N t^{\gamma}
$$

for some constants $N$ and $\gamma>0$ depending only on $a, b$ and $\omega_{1}$. By Fubini's theorem, we also get $\int_{0}^{1} \tilde{\omega}(t) / t d t<\infty$. The lemma is proved.

We will also need the following energy inequality and a variant of the parabolic Poincaré inequality.

Lemma 3.2. Let $B=\hat{B}=C=0$ and $R>0$. Suppose $v \in \mathcal{H}_{2}^{1}\left(Q_{R}\right)$ is a weak solution to the equation

$$
\left\{\begin{aligned}
\mathcal{P} v=\operatorname{div} g+f & \text { in } Q_{R} \\
v=0 & \text { on } \partial_{p} Q_{R}
\end{aligned}\right.
$$

where $f, g \in L_{2}\left(Q_{R}\right)$. Then we have

$$
\|D v\|_{L_{2}\left(Q_{R}\right)}+R^{-1}\|v\|_{L_{2}\left(Q_{R}\right)} \leq N\|g\|_{L_{2}\left(Q_{R}\right)}+N R\|f\|_{L_{2}\left(Q_{R}\right)},
$$

where $N=N(d, n, \nu)>0$.

Lemma 3.3. Let $p \in(1, \infty), r \in(0, \infty)$ and $u \in C_{l o c}^{\infty}\left(\mathbb{R}^{d+1}\right)$.

i) Suppose $B=\hat{B}=C=0, g, f \in L_{p, l o c}$, and $\mathcal{P} u=\operatorname{div} g+f$ in $Q_{r}$. Then

$$
\int_{Q_{r}}\left|u(t, x)-(u)_{Q_{r}}\right|^{p} d z \leq N r^{p} \int_{Q_{r}}\left(|D u|^{p}+|g|^{p}+r^{p}|f|^{p}\right) d z,
$$

where $N=N(d, \nu, p)>0$. 
ii) There is a constant $N=N(d, p)$ such that

$$
\begin{array}{r}
\int_{Q_{r}}\left|D u(t, x)-(D u)_{Q_{r}}\right|^{p} d z \leq N r^{p} \int_{Q_{r}}\left(\left|D^{2} u\right|^{p}+\left|u_{t}\right|^{p}\right) d z \\
\int_{Q_{r}}\left|u(t, x)-(u)_{Q_{r}}-x^{\beta}\left(D_{\beta} u\right)_{Q_{r}}\right|^{p} d z \leq N r^{2 p} \int_{Q_{r}}\left(\left|D^{2} u\right|^{p}+\left|u_{t}\right|^{p}\right) d z .
\end{array}
$$

Proof. See, for instance, Lemma 3.1 and Lemma 3.2 of [12]. We remark that by an approximation argument, (3.2) remains valid for $u \in \mathcal{H}_{p, \text { loc }}^{1}$, and (3.3) remains valid for $u \in W_{p, l o c}^{1,2}$.

In the remaining part of the section, we shall prove some local estimates, which are deduced from the results obtained in [4].

Lemma 3.4. Let $p \in(1, \infty)$. Assume $A^{\alpha \beta}$ are partially VMO in $z^{\prime}, u \in$ $\mathcal{H}_{p}^{1}\left(Q_{1}\right)$ and

$$
\mathcal{P} u=\operatorname{div} g+f,
$$

in $Q_{1}$, where $f, g \in L_{p}\left(Q_{1}\right)$. Then there exists a constant $N=N\left(d, n, \nu, K, \omega_{A, z^{\prime}}, p\right)$ such that

$$
\|u\|_{\mathcal{H}_{p}^{1}\left(Q_{1 / 2}\right)} \leq N\left(\|u\|_{L_{p}\left(Q_{1}\right)}+\|g\|_{L_{p}\left(Q_{1}\right)}+\|f\|_{L_{p}\left(Q_{1}\right)}\right) .
$$

Proof. The lemma follows from Theorem 2.2 of 4 by a standard localization argument. For the sake of completeness, we give a proof in the Appendix.

By using the Sobolev imbedding theorem and a bootstrap argument, we get

Corollary 3.5. Let $p, q \in(1, \infty)$. Assume $A^{\alpha \beta}$ are partially VMO in $z^{\prime}$, $u \in C_{\text {loc }}^{\infty}$ satisfies (3.4) in $Q_{1}$, where $f, g \in L_{q}\left(Q_{1}\right)$. Then there exists a constant $N=N\left(d, n, \nu, K, \omega_{A, z^{\prime}}, p, q\right)$ such that

$$
\|u\|_{\mathcal{H}_{q}^{1}\left(Q_{1 / 2}\right)} \leq N\left(\|u\|_{L_{p}\left(Q_{1}\right)}+\|g\|_{L_{q}\left(Q_{1}\right)}+\|f\|_{L_{q}\left(Q_{1}\right)}\right) .
$$

In particular, if $q>d+2$, it holds that

$$
|u|_{\gamma / 2, \gamma ; Q_{1 / 2}} \leq N\left(\|u\|_{L_{p}\left(Q_{1}\right)}+\|g\|_{L_{q}\left(Q_{1}\right)}+\|f\|_{L_{q}\left(Q_{1}\right)}\right),
$$

where $\gamma=1-(d+2) / q$.

Next we consider systems with coefficients depending on $x^{d}$ alone. We denote

$$
\mathcal{P}_{0} u=-u_{t}+D_{\alpha}\left(A^{\alpha \beta}\left(x^{d}\right) D_{\beta} u\right),
$$

and

$$
U:=A^{d \beta} D_{\beta} u, \quad \text { i.e., } \quad U^{i}=A_{i j}^{d \beta} D_{\beta} u^{j}, \quad i=1, \cdots, n .
$$


Lemma 3.6. Let $p \in(1, \infty)$. Assume $u \in C_{l o c}^{\infty}$ satisfies $\mathcal{P}_{0} u=0$ in $Q_{1}$. Then for any nonnegative integers $i, j$ such that $i+j \geq 1$ and any $q \in(1, \infty)$, there exists a constant $N=N(d, n, p, q, i, j, \nu)$ such that

$$
\left\|D_{t}^{i} D_{x^{\prime}}^{j} u\right\|_{\mathcal{H}_{q}^{1}\left(Q_{1 / 2}\right)} \leq N\|D u\|_{L_{p}\left(Q_{1}\right)} .
$$

For any $\gamma \in(0,1)$, we also have

$$
\left|D_{t}^{i} D_{x^{\prime}}^{j} u\right|_{\gamma / 2, \gamma ; Q_{1 / 2}} \leq N\|D u\|_{L_{p}\left(Q_{1}\right)} .
$$

Moreover,

$$
\begin{aligned}
\left\|D_{t}^{i} D_{x^{\prime}}^{j} U\right\|_{\mathcal{H}_{q}^{1}\left(Q_{1 / 2}\right)} & \leq N\|D u\|_{L_{p}\left(Q_{1}\right)}, \\
\left|D_{t}^{i} D_{x^{\prime}}^{j} U\right|_{\gamma / 2, \gamma ; Q_{1 / 2}} & \leq N\|D u\|_{L_{p}\left(Q_{1}\right)} .
\end{aligned}
$$

Proof. Note that $\mathcal{P}_{0}\left(D_{t}^{i} D_{x^{\prime}}^{j} u\right)=0$ in $Q_{1}$. Thanks to Lemma 3.4 and Corollary [3.5, to prove (3.5) it suffices to show that for any $1 / 2 \leq r<R \leq 1$,

$$
\left\|u_{t}\right\|_{L_{2}\left(Q_{r}\right)} \leq N(d, n, \nu, r, R)\|D u\|_{L_{2}\left(Q_{R}\right)} .
$$

Indeed, if $i \geq 1$, by Lemma 3.4 and (3.9), for any $1 / 2 \leq r_{1}<r_{2}<r_{3} \leq 1$,

$$
\left\|D_{t}^{i} D_{x^{\prime}}^{j} u\right\|_{\mathcal{H}_{q}^{1}\left(Q_{r_{1}}\right)} \leq N\left\|D_{t}^{i} D_{x^{\prime}}^{j} u\right\|_{L_{2}\left(Q_{r_{2}}\right)} \leq N\left\|D_{t}^{i-1} D_{x^{\prime}}^{j} u\right\|_{\mathcal{H}_{2}^{1}\left(Q_{r_{3}}\right)} .
$$

Similarly, if $j \geq 1$, we have

$$
\left\|D_{t}^{i} D_{x^{\prime}}^{j} u\right\|_{\mathcal{H}_{q}^{1}\left(Q_{r_{1}}\right)} \leq N\left\|D_{t}^{i} D_{x^{\prime}}^{j} u\right\|_{L_{2}\left(Q_{r_{2}}\right)} \leq N\left\|D_{t}^{i} D_{x^{\prime}}^{j-1} u\right\|_{\mathcal{H}_{2}^{1}\left(Q_{r_{3}}\right)} .
$$

Repeating this procedure to reduce $i$ and $j$, we reach

$$
\left\|D_{t}^{i} D_{x^{\prime}}^{j} u\right\|_{\mathcal{H}_{q}^{1,2}\left(Q_{1 / 2}\right)} \leq N\left\|u_{t}\right\|_{L_{2}\left(Q_{3 / 4}\right)}+N\|D u\|_{L_{2}\left(Q_{3 / 4}\right)} \leq N\|D u\|_{L_{2}\left(Q_{1}\right)} .
$$

In the last inequality, we used (3.9). For the proof of (3.9), see for instance Lemma 3.3 of [4]. Inequality (3.6) is deduced from (3.5) by the parabolic Sobolev imbedding theorem.

To prove (3.7) and (3.8), one only needs to observe that in $Q_{1}$,

$$
D_{d} U=u_{t}-\sum_{\alpha=1}^{d-1} \sum_{\beta=1}^{d} D_{\alpha}\left(A^{\alpha \beta} D_{\beta} u\right)=u_{t}-\sum_{\alpha=1}^{d-1} \sum_{\beta=1}^{d} A^{\alpha \beta} D_{\alpha \beta} u
$$

and

$$
D_{x^{\prime}} U=\sum_{\beta=1}^{d} A^{d \beta} D_{x^{\prime}} D_{\beta} u, \quad D_{t} U=\sum_{\beta=1}^{d} A^{d \beta} D_{t} D_{\beta} u .
$$

The $L_{p}$ norms of the right-hand sides are bounded by $\|D u\|_{L_{p}}$ due to (3.5). 


\section{Systems with Partially Dini Coefficients}

This section is devoted to the proof of Theorem 2.1. The following lemma reduces the estimate of $[u]_{1 / 2,1}$ to the estimate of $|D u|_{0}$.

Lemma 4.1. Let $u$ be a weak solution to (2.1) in $Q_{1}$. Suppose $|u|_{0 ; Q_{1 / 2}}<\infty$ and $|D u|_{0 ; Q_{1 / 2}}<\infty$. Then we have

$$
[u]_{1 / 2,1 ; Q_{1 / 4}} \leq N\left(|u|_{0 ; Q_{1 / 2}}+|D u|_{0 ; Q_{1 / 2}}+|f|_{0 ; Q_{1 / 2}}+|g|_{0 ; Q_{1 / 2}}\right) .
$$

Proof. Rewrite (2.1) as

$$
-u_{t}+D_{\alpha}\left(A^{\alpha \beta} D_{\beta} u\right)=D_{\alpha}\left(g_{\alpha}-B^{\alpha} u\right)+f-\hat{B}^{\alpha} D_{\alpha} u-C u:=D_{\alpha} \mathfrak{g}_{\alpha}+\mathfrak{f},
$$

where

$$
\mathfrak{g}_{\alpha}=g_{\alpha}-B^{\alpha} u, \quad \mathfrak{f}=f-\hat{B}^{\alpha} D_{\alpha} u-C u .
$$

We fix $z_{0} \in \overline{Q_{1 / 4}}$ and take $r \in(0,1 / 4)$. By Lemma 3.3, we have

$$
\begin{gathered}
\int_{Q_{r}\left(z_{0}\right)}\left|u-(u)_{Q_{r}\left(z_{0}\right)}\right|^{2} d z \leq N r^{2} \int_{Q_{r}\left(z_{0}\right)}\left(|D u|^{2}+|\mathfrak{g}|^{2}+r^{2}|\mathfrak{f}|^{2}\right) \\
\leq N r^{n+4}\left(|D u|_{0 ; Q_{1 / 2}}+|u|_{0 ; Q_{1 / 2}}+|g|_{0 ; Q_{1 / 2}}+|f|_{0 ; Q_{1 / 2}}\right)^{2} .
\end{gathered}
$$

The inequality (4.1) then follows from Campanato's characterization of Hölder continuous functions; see, for instance, [17, Lemma 4.3].

Now we are ready to prove Theorem 2.1. We shall divide the proof into two steps.

Step 1: Estimate of $|D u|_{0}$. We first assume that all the coefficients and data are smooth, so that by the classical theory $|D u|_{0 ; Q_{3 / 4}}<\infty$. We take $0<\gamma_{1}<\gamma<1$. Fix a point $z_{0} \in Q_{3 / 4}$, and take $0<r<R \leq\left(3 / 4-\left|x_{0}\right|\right) / 2$. Now take $z_{1}^{\prime} \in Q_{R}^{\prime}\left(z_{0}^{\prime}\right)$ and denote

$$
\mathcal{P}_{z_{1}^{\prime}} u:=-u_{t}+D_{\alpha}\left(A^{\alpha \beta}\left(z_{1}^{\prime}, x^{d}\right) D_{\beta} u\right) .
$$

Recall the definitions of $\mathfrak{g}$ and $\mathfrak{f}$ in (4.2). Then we have

$$
\mathcal{P}_{z_{1}^{\prime}} u=\operatorname{div}(\mathfrak{g}+m)+\mathfrak{f},
$$

where

$$
m_{\alpha}^{i}(z)=\left(A_{i j}^{\alpha \beta}\left(z_{1}^{\prime}, x^{d}\right)-A_{i j}^{\alpha \beta}(z)\right) D_{\beta} u^{j}
$$

Let

$$
u_{0}\left(x^{d}\right)=\int_{-1}^{x^{d}}\left(A^{d d}\left(z_{1}^{\prime}, s\right)\right)^{-1} \mathfrak{g}_{d}\left(z_{1}^{\prime}, s\right) d s, \quad u_{e}=u-u_{0}
$$

Clearly,

$$
\mathcal{P}_{z_{1}^{\prime}} u_{e}=\operatorname{div}\left(\mathfrak{g}(z)-\mathfrak{g}\left(z_{1}^{\prime}, x^{d}\right)+m(z)\right)+\mathfrak{f} .
$$

Let $v$ be a weak solution to the equation

$$
\left\{\begin{aligned}
\mathcal{P}_{z_{1}^{\prime}} v=\operatorname{div}\left(\mathfrak{g}(z)-\mathfrak{g}\left(z_{0}^{\prime}, x^{d}\right)+m(z)\right)+\mathfrak{f} & \text { in } Q_{R}\left(z_{0}\right) \\
v=0 & \text { on } \partial_{p} Q_{R}\left(z_{0}\right) .
\end{aligned}\right.
$$


By Lemma 3.2, we get

$$
\begin{aligned}
& \|D v\|_{L_{2}\left(Q_{R}\left(z_{0}\right)\right)} \\
& \leq N\left\|\mathfrak{g}(z)-\mathfrak{g}\left(z_{1}^{\prime}, x^{d}\right)+m\right\|_{L_{2}\left(Q_{R}\left(z_{0}\right)\right)}+N R\|\mathfrak{f}\|_{L_{2}\left(Q_{R}\left(z_{0}\right)\right)} \\
& \leq N\left\|\mathfrak{g}(z)-\mathfrak{g}\left(z_{1}^{\prime}, x^{d}\right)+m\right\|_{L_{2}\left(Q_{R}\left(z_{0}\right)\right)}+N|\mathfrak{f}|_{0 ; Q_{R}\left(z_{0}\right)} R^{d / 2+1+\gamma_{1}} .
\end{aligned}
$$

Let $w=u_{e}-v$. Then $w$ satisfies $\mathcal{P}_{z_{1}^{\prime}} w=0$ in $Q_{R}\left(z_{0}\right)$. Denote

$$
W:=A^{d \beta}\left(z_{1}^{\prime}, x^{d}\right) D_{\beta} w .
$$

It follows from Lemma 3.6 and a suitable scaling that

$$
\begin{aligned}
& \int_{Q_{r}\left(z_{0}\right)}\left|D_{x^{\prime}} w-\left(D_{x^{\prime}} w\right)_{Q_{r}\left(z_{0}\right)}\right|^{2}+\left|W-(W)_{Q_{r}\left(z_{0}\right)}\right|^{2} d z \\
& \leq N(r / R)^{d+2+2 \gamma} \int_{Q_{R}\left(z_{0}\right)}|D w|^{2} d z \\
& \leq N(r / R)^{d+2+2 \gamma} \int_{Q_{R}\left(z_{0}\right)}\left|D_{x^{\prime}} w\right|^{2}+|W|^{2} d z .
\end{aligned}
$$

In the last inequality, we also used the nondegeneracy of $A^{d d}$. Define

$$
h\left(x^{d}\right)=\int_{0}^{x^{d}}\left(A^{d d}\left(z_{1}^{\prime}, s\right)\right)^{-1}\left((W)_{Q_{R}\left(z_{0}\right)}-\sum_{\beta=1}^{d-1} A^{d \beta}\left(z_{1}^{\prime}, \cdot\right)\left(D_{\beta} w\right)_{Q_{R}\left(z_{0}\right)}\right) d s,
$$

and

$$
\tilde{w}:=w-\sum_{\beta=1}^{d-1} x^{\beta}\left(D_{\beta} w\right)_{Q_{R}\left(z_{0}\right)}-h\left(x^{d}\right) .
$$

We define $\tilde{W}$ and $U_{e}$ as in (4.4) with $\tilde{w}$ and $u_{e}$ in place of $w$ respectively. Then,

$$
D_{x^{\prime}} \tilde{w}=D_{x^{\prime}} w-\left(D_{x^{\prime}} w\right)_{Q_{R}\left(z_{0}\right)}, \quad \tilde{W}=W-(W)_{Q_{R}\left(z_{0}\right)} .
$$

A direct calculation shows that $\tilde{w}$ also satisfies $\mathcal{P}_{z_{1}^{\prime}} \tilde{w}=0$ in $Q_{R}\left(z_{0}\right)$. We substitute $w$ and $W$ in (4.5) by $\tilde{w}$ and $\tilde{W}$ to get

$$
\begin{aligned}
& \int_{Q_{r}\left(z_{0}\right)}\left|D_{x^{\prime}} w-\left(D_{x^{\prime}} w\right)_{Q_{r}\left(z_{0}\right)}\right|^{2}+\left|W-(W)_{Q_{r}\left(z_{0}\right)}\right|^{2} d z \\
& \leq N(r / R)^{d+2+2 \gamma} \int_{Q_{R}\left(z_{0}\right)}\left|D_{x^{\prime}} w-\left(D_{x^{\prime}} w\right)_{Q_{R}\left(z_{0}\right)}\right|^{2}+\left|W-(W)_{Q_{R}\left(z_{0}\right)}\right|^{2} d z .
\end{aligned}
$$

We combine (4.3) with (4.6) and use the triangle inequality to obtain

$$
\begin{aligned}
& \int_{Q_{r}\left(z_{0}\right)}\left|D_{x^{\prime}} u_{e}-\left(D_{x^{\prime}} u_{e}\right)_{Q_{r}\left(z_{0}\right)}\right|^{2}+\left|U_{e}-\left(U_{e}\right)_{Q_{r}\left(z_{0}\right)}\right|^{2} d z \\
& \leq N_{1}(r / R)^{d+2+2 \gamma} \int_{Q_{R}\left(z_{0}\right)}\left|D_{x^{\prime}} u_{e}-\left(D_{x^{\prime}} u_{e}\right)_{Q_{R}\left(z_{0}\right)}\right|^{2}+\left|U_{e}-\left(U_{e}\right)_{Q_{R}\left(z_{0}\right)}\right|^{2} d z \\
& +N\left\|\mathfrak{g}(z)-\mathfrak{g}\left(z_{1}^{\prime}, x^{d}\right)+m\right\|_{L_{2}\left(Q_{R}\left(z_{0}\right)\right)}^{2}+N|\mathfrak{f}|_{0 ; Q_{R}\left(z_{0}\right)}^{2} R^{d+2+2 \gamma_{1}} .
\end{aligned}
$$


where $N_{1}=N_{1}(d, n, \nu)$. It is not very convenient to use (4.7) since both $u_{e}$ and $U_{e}$ depend on $z_{0}$. However, by the definition of $u_{0}, u_{e}$ and $U_{e}$,

$$
D_{x^{\prime}} u_{e}=D_{x^{\prime}} u, \quad U_{e}=A^{d \beta}\left(z_{1}^{\prime}, x^{d}\right) D_{\beta} u-\mathfrak{g}_{d}\left(z_{1}^{\prime}, x^{d}\right)
$$

Recall that

$$
\hat{U}=A^{d \beta} D_{\beta} u-\mathfrak{g}_{d}(z),
$$

which is independent of the choice of $z_{0}$. Clearly, in $Q_{R}\left(z_{0}\right)$

$$
\left|\hat{U}(z)-U_{e}(z)\right| \leq N\left|A(z)-A\left(z_{1}^{\prime}, x^{d}\right)\right||D u|_{0 ; Q_{R}\left(z_{0}\right)}+\left|\mathfrak{g}_{d}(z)-\mathfrak{g}_{d}\left(z_{1}^{\prime}, x^{d}\right)\right| .
$$

Thus, coming back to (4.7) we get

$$
\begin{aligned}
& \int_{Q_{r}\left(z_{0}\right)}\left|D_{x^{\prime}} u-\left(D_{x^{\prime}} u\right)_{Q_{r}\left(z_{0}\right)}\right|^{2}+\left|\hat{U}-(\hat{U})_{Q_{r}\left(z_{0}\right)}\right|^{2} d z \\
& \leq N_{1}(r / R)^{d+2+2 \gamma} \int_{Q_{R}\left(z_{0}\right)}\left|D_{x^{\prime}} u-\left(D_{x^{\prime}} u\right)_{Q_{R}\left(z_{0}\right)}\right|^{2}+\left|\hat{U}-(\hat{U})_{Q_{R}\left(z_{0}\right)}\right|^{2} d z \\
& +N\left\|\mathfrak{g}(z)-\mathfrak{g}\left(z_{1}^{\prime}, x^{d}\right)\right\|_{L_{2}\left(Q_{R}\left(z_{0}\right)\right)}^{2}+N\left\|A(z)-A\left(z_{1}^{\prime}, x^{d}\right)\right\|_{L_{2}\left(Q_{R}\left(z_{0}\right)\right)}^{2}|D u|_{0 ; Q_{R}\left(z_{0}\right)}^{2} \\
& +N|\mathfrak{f}|_{0 ; Q_{R}\left(z_{0}\right)}^{2} R^{d+2+2 \gamma_{1}} .
\end{aligned}
$$

Now we take the average of both sides of (4.9) with respect to $z_{1}^{\prime} \in Q_{R}^{\prime}\left(z_{0}\right)$, and use the definitions of $\mathfrak{g}$ and $\mathfrak{f}$ to obtain

$$
\begin{aligned}
& \int_{Q_{r}\left(z_{0}\right)}\left|D_{x^{\prime}} u-\left(D_{x^{\prime}} u\right)_{Q_{r}\left(z_{0}\right)}\right|^{2}+\left|\hat{U}-(\hat{U})_{Q_{r}\left(z_{0}\right)}\right|^{2} d z \\
& \leq N_{1}(r / R)^{d+2+2 \gamma} \int_{Q_{R}\left(z_{0}\right)}\left|D_{x^{\prime}} u-\left(D_{x^{\prime}} u\right)_{Q_{R}\left(z_{0}\right)}\right|^{2}+\left|\hat{U}-(\hat{U})_{Q_{R}\left(z_{0}\right)}\right|^{2} d z \\
& +N\left(\omega_{g, z^{\prime}}(R)+\omega_{B, z^{\prime}}(R)|u|_{0 ; Q_{3 / 4}}+\omega_{A, z^{\prime}}(R)|D u|_{0 ; Q_{R}\left(z_{0}\right)}\right)^{2} R^{d+2} \\
& +N\left([u]_{\gamma_{1} / 2, \gamma_{1} ; Q_{3 / 4}}+|\mathfrak{f}|_{0 ; Q_{R}\left(z_{0}\right)}\right)^{2} R^{d+2+2 \gamma_{1}} .
\end{aligned}
$$

Set $r=\tau R$ for some $\tau \in(0,1)$ to be chosen later, and denote

$$
\phi_{r}\left(z_{0}\right)=f_{Q_{r}\left(z_{0}\right)}\left|D_{x^{\prime}} u-\left(D_{x^{\prime}} u\right)_{Q_{r}\left(z_{0}\right)}\right|^{2}+\left|\hat{U}-(\hat{U})_{Q_{r}\left(z_{0}\right)}\right|^{2} d z .
$$

It follows from (4.10) that

$$
\begin{gathered}
\phi_{\tau R}\left(z_{0}\right) \leq N_{1} \tau^{2 \gamma} \phi_{R}\left(z_{0}\right)+N\left(\omega_{g, z^{\prime}}^{2}(R)+\omega_{B, z^{\prime}}^{2}(R)|u|_{0 ; Q_{3 / 4}}^{2}\right) \tau^{-d-2} \\
+N \omega_{A, z^{\prime}}^{2}(R)|D u|_{0 ; Q_{R}\left(z_{0}\right)}^{2} \tau^{-d-2}+N\left([u]_{\gamma_{1} / 2, \gamma_{1} ; Q_{3 / 4}}^{2}+|\mathfrak{f}|_{0 ; Q_{R}\left(z_{0}\right)}^{2}\right) \tau^{-d-2} R^{2 \gamma_{1}} .
\end{gathered}
$$

We fix $\tau=\tau(d, n, \nu)<1$ sufficiently small such that $N_{1} \tau^{2 \gamma} \leq 1 / 2$. By an iteration, we obtain,

$$
\phi_{\tau^{k} R}\left(z_{0}\right) \leq 2^{-k} \phi_{R}\left(z_{0}\right)+N \sum_{j=1}^{k} 2^{-j}\left(\omega_{g, z^{\prime}}^{2}\left(\tau^{k-j} R\right)+\omega_{B, z^{\prime}}^{2}\left(\tau^{k-j} R\right)|u|_{0 ; Q_{3 / 4}}^{2}\right.
$$


$\left.+\omega_{A, z^{\prime}}^{2}\left(\tau^{k-j} R\right)|D u|_{0 ; Q_{R}\left(z_{0}\right)}^{2}\right)+N\left([u]_{\gamma_{1} / 2, \gamma_{1} ; Q_{3 / 4}}+|\mathfrak{f}|_{0 ; Q_{R}\left(z_{0}\right)}\right)^{2} \sum_{j=1}^{k} 2^{-j}\left(\tau^{k-j} R\right)^{2 \gamma_{1}}$.

We define

$$
\psi_{r}\left(z_{0}\right)=f_{Q_{r}\left(z_{0}\right)}\left|D_{x^{\prime}} u\right|+|\hat{U}| d z .
$$

By the triangle inequality and Hölder's inequality,

$$
\begin{aligned}
\left|\psi_{\tau r}\left(z_{0}\right)-\psi_{r}\left(z_{0}\right)\right| & \leq f_{Q_{\tau r}\left(z_{0}\right)}\left|D_{x^{\prime}} u-\left(D_{x^{\prime}} u\right)_{Q_{r}\left(z_{0}\right)}\right|+\left|\hat{U}-(\hat{U})_{Q_{r}\left(z_{0}\right)}\right| d z \\
& \leq N\left(\phi_{r}\left(z_{0}\right)\right)^{1 / 2}
\end{aligned}
$$

Combining (4.11) and (4.12), we deduce

$$
\begin{gathered}
\left|\psi_{\tau^{k} R}\left(z_{0}\right)-\psi_{\tau^{k-1} R}\left(z_{0}\right)\right| \leq N 2^{-k / 2} \phi_{R}^{1 / 2}\left(z_{0}\right) \\
+N \sum_{j=1}^{k} 2^{-j / 2}\left(\omega_{g, z^{\prime}}\left(\tau^{k-j} R\right)+\omega_{B, z^{\prime}}\left(\tau^{k-j} R\right)|u|_{0 ; Q_{3 / 4}}+\omega_{A, z^{\prime}}\left(\tau^{k-j} R\right)|D u|_{0 ; Q_{R}\left(z_{0}\right)}\right) \\
+N\left([u]_{\gamma_{1} / 2, \gamma_{1} ; Q_{3 / 4}}+|\mathfrak{f}|_{0 ; Q_{R}\left(z_{0}\right)}\right) \sum_{j=1}^{k} 2^{-j / 2}\left(\tau^{k-j} R\right)^{\gamma_{1}} .
\end{gathered}
$$

Summing the inequality above in $k$ gives

$$
\begin{gathered}
\psi_{\tau^{k} R}\left(z_{0}\right) \leq \psi_{R}\left(z_{0}\right)+N \phi_{R}^{1 / 2}\left(z_{0}\right)+N \sum_{j=1}^{k}\left(\omega_{g, z^{\prime}}\left(\tau^{k-j} R\right)+\omega_{B, z^{\prime}}\left(\tau^{k-j} R\right)|u|_{0 ; Q_{3 / 4}}\right) \\
\quad+N \sum_{j=1}^{k} \omega_{A, z^{\prime}}\left(\tau^{k-j} R\right)|D u|_{0 ; Q_{R}\left(z_{0}\right)}+N\left([u]_{\gamma_{1} / 2, \gamma_{1} ; Q_{3 / 4}}+|\mathfrak{f}|_{0 ; Q_{R}\left(z_{0}\right)}\right) R^{\gamma_{1}},
\end{gathered}
$$

where $N=N(d, n, \nu)$. To estimate the summations on the right-hand side, we recall

$$
I[\omega](r)=\int_{0}^{r} \omega(s) / s d s .
$$

It is easy to see that for any Dini function $\omega$ and $k \geq 1$,

$$
\sum_{j=1}^{k} \omega\left(\tau^{k-j} R\right) \leq N I[\omega]\left(\tau^{-1} R\right)
$$

Therefore, we deduce

$$
\begin{gathered}
\psi_{\tau^{k} R}\left(z_{0}\right) \leq \psi_{R}\left(z_{0}\right)+N \phi_{R}^{1 / 2}\left(z_{0}\right)+N I\left[\omega_{g, z^{\prime}}\right]\left(\tau^{-1} R\right)+N I\left[\omega_{B, z^{\prime}}\right]\left(\tau^{-1} R\right)|u|_{0 ; Q_{3 / 4}} \\
+N I\left[\omega_{A, z^{\prime}}\right]\left(\tau^{-1} R\right)|D u|_{0 ; Q_{R}\left(z_{0}\right)}+N\left([u]_{\gamma_{1} / 2, \gamma_{1} ; Q_{3 / 4}}+|\mathfrak{f}|_{0 ; Q_{R}\left(z_{0}\right)}\right) R^{\gamma_{1}}
\end{gathered}
$$


It follows from (4.13) and the definition of $\mathfrak{f}$ that

$$
\begin{aligned}
& (|D u|)_{Q_{\tau^{k}}\left(z_{0}\right)} \leq \psi_{R}\left(z_{0}\right)+N \phi_{R}^{1 / 2}\left(z_{0}\right)+N I\left[\omega_{g, z^{\prime}}\right]\left(\tau^{-1} R\right) \\
& \quad+N I\left[\omega_{B, z^{\prime}}\right]\left(\tau^{-1} R\right)|u|_{0 ; Q_{3 / 4}}+N\left(|g|_{0 ; Q_{3 / 4}}+|u|_{0 ; Q_{3 / 4}}+[u]_{\gamma_{1} / 2, \gamma_{1} ; Q_{3 / 4}}\right) \\
& \quad+N_{2}\left(I\left[\omega_{A, z^{\prime}}\right]\left(\tau^{-1} R\right)+R^{\gamma_{1}}\right)|D u|_{0 ; Q_{R}\left(z_{0}\right)}+N|f|_{0 ; Q_{R}\left(z_{0}\right)} R^{\gamma_{1}},
\end{aligned}
$$

where $N_{2}=N_{2}(d, n, \nu)>0$. We choose $\left.R_{0}=R_{0}\left(d, n, \nu, \omega_{A, z^{\prime}}\right) \in(0, \tau / 4)\right)$ such that

$$
N_{2}\left(I\left[\omega_{A, z^{\prime}}\right]\left(\tau^{-1} R_{0}\right)+R_{0}^{\gamma_{1}}\right)<2^{-d-2} .
$$

Now we confine $z_{0}$ to $Q^{(\ell)}:=Q_{3 / 4-2^{-\ell-1} R_{0}}, \ell=1,2, \ldots$ and set $R=2^{-\ell-2} R_{0}$. Since $k$ and $z_{0} \in Q^{(\ell)}$ in (4.14) are arbitrary, due to the Lebesgue lemma, we obtain

$$
\begin{aligned}
& |D u|_{0 ; Q^{(\ell)}} \leq N_{3} 2^{\ell(d+2) / 2}\left(\|D u\|_{L_{2}\left(Q_{1}\right)}+|g|_{0 ; Q_{3 / 4}}+I\left[\omega_{g, z^{\prime}}\right](1)+|u|_{0 ; Q_{3 / 4}}\right) \\
& \quad+N_{3} 2^{\ell(d+2) / 2}\left(|f|_{0 ; Q_{R}\left(z_{0}\right)}+[u]_{\gamma_{1} / 2, \gamma_{1} ; Q_{3 / 4}}\right)+2^{-d-2}|D u|_{0 ; Q^{(\ell+1)}},
\end{aligned}
$$

where $N_{3}=N_{3}\left(d, n, \nu, \omega_{A, z^{\prime}}, \omega_{B, z^{\prime}}\right)>0$. Multiplying both sides of (4.15) by $2^{-\ell(d+2)}$ and summing in $\ell=1,2, \ldots$ yield

$$
\begin{gathered}
\sum_{\ell=1}^{\infty} 2^{-\ell(d+2)}|D u|_{0 ; Q^{(\ell)}} \leq N_{3}\left(\|D u\|_{L_{2}\left(Q_{1}\right)}+|g|_{0 ; Q_{3 / 4}}+I\left[\omega_{g, z^{\prime}}\right](1)+|u|_{0 ; Q_{3 / 4}}\right) \\
+N_{3}\left(|f|_{0 ; Q_{R}\left(z_{0}\right)}+[u]_{\gamma_{1} / 2, \gamma_{1} ; Q_{3 / 4}}\right)+\sum_{\ell=1}^{\infty} 2^{-(\ell+1)(d+2)}|D u|_{0 ; Q^{(\ell+1)}} .
\end{gathered}
$$

Since we assume $|D u|_{0 ; Q_{3 / 4}}<\infty$, it follows from the inequality above by absorbing the summation on the right-hand side into the left-hand side that

$$
\begin{aligned}
|D u|_{0 ; Q^{(1)}} \leq N_{3} & \left(\|D u\|_{L_{2}\left(Q_{1}\right)}+|g|_{0 ; Q_{3 / 4}}+I\left[\omega_{g, z^{\prime}}\right](1)+|u|_{0 ; Q_{3 / 4}}\right) \\
& +N_{3}\left(|f|_{0 ; Q_{R}\left(z_{0}\right)}+[u]_{\gamma_{1} / 2, \gamma_{1} ; Q_{3 / 4}}\right) .
\end{aligned}
$$

To finish the proof of (2.3), it suffices to use Corollary 3.5 .

Now we remove the smoothness assumption on the coefficients and data by using a standard approximation argument, which we sketched below for the completeness. Let $A_{(m)}^{\alpha \beta}, m=1,2, \ldots$ be the mollifications of $A^{\alpha \beta}$. Similarly, we define $B_{(m)}^{\alpha}, \hat{B}_{(m)}^{\alpha}, C_{(m)}^{\alpha}, g_{(m)}$ and $f_{(m)}$. We know that $A_{(m)}^{\alpha \beta}, m=1,2, \ldots$ are uniformly elliptic with the same ellipticity constant $\nu$, and as $m \rightarrow \infty$,

$$
\begin{aligned}
\left(f_{(m)}, g_{(m)}\right) & \rightarrow(f, g) \text { in } L_{2}\left(Q_{1}\right), \\
\left(A_{(m)}^{\alpha \beta}, B_{(m)}^{\alpha}, \hat{B}_{(m)}^{\alpha}\right) & \rightarrow\left(A^{\alpha \beta}, B^{\alpha}, \hat{B}^{\alpha}\right) \text { a.e.. }
\end{aligned}
$$

Moreover,

$$
\omega_{A_{(m)}, z^{\prime}} \leq \omega_{A, z^{\prime}}, \quad \omega_{B_{(m)}, z^{\prime}} \leq \omega_{B, z^{\prime}}
$$


Let $\mathcal{P}_{m}$ be the parabolic operator with coefficients $A_{(m)}^{\alpha \beta}, B_{(m)}^{\alpha}$ and $C_{(m)}$ instead of $A^{\alpha \beta}, B^{\alpha}$ and $C$. Let $v_{m} \in \mathcal{H}_{2}^{1}\left(Q_{1}\right)$ be the weak solution to the equation

$$
\begin{aligned}
\mathcal{P}_{m} v_{m}=\operatorname{div}( & \left.g-g_{(m)}\right)+f-f_{(m)}+D_{\alpha}\left(\left(A_{(m)}^{\alpha \beta}-A^{\alpha \beta}\right) D_{\beta} u\right. \\
& \left.+\left(B_{(m)}^{\alpha}-B^{\alpha}\right) u\right)+\left(\hat{B}_{(m)}^{\alpha}-\hat{B}^{\alpha}\right) D_{\alpha} u+\left(\hat{C}_{(m)}-\hat{C}\right) u
\end{aligned}
$$

in $Q_{1}$ with the zero initial-boundary condition $v_{m}=0$ on the parabolic boundary of $Q_{1}$. Note that the right-hand side of (4.16) goes to zero in $L_{2}$ as $m \rightarrow \infty$. It follows from the $L_{2}$ estimate that $\left\|v_{m}\right\|_{L_{2}\left(Q_{1}\right)}+\left\|D v_{m}\right\|_{L_{2}\left(Q_{1}\right)} \rightarrow 0$ as $m \rightarrow \infty$, which further implies that there is subsequence, which is still denoted by $\left\{v_{m}\right\}$, such that $\left|D v_{m}\right|+\left|v_{m}\right| \rightarrow 0$ a.e. in $Q_{1}$. Let $u_{m}=u-v_{m}$. It is clear that $u_{m}$ is a weak solution to the equation

$$
\mathcal{P}_{m} u_{m}=\operatorname{div} g_{(m)}+f_{(m)}
$$

in $Q_{1}$. By the classical parabolic theory, $u_{m}$ is infinitely differentiable in $Q_{1}$. Thus, by the proof above and by slightly shrinking the domain, we obtain a uniform estimate

$$
\begin{aligned}
\left|D u_{m}\right|_{0 ; Q_{1 / 2}} & \leq N\left(I\left[\omega_{g, z^{\prime}}\right](1)+\left|g_{(m)}\right|_{0 ; Q_{5 / 6}}+\left|f_{(m)}\right|_{0 ; Q_{5 / 6}}+\left\|u_{m}\right\|_{L_{2}\left(Q_{5 / 6}\right)}\right) \\
& \leq N\left(I\left[\omega_{g, z^{\prime}}\right](1)+|g|_{0 ; Q_{1}}+|f|_{0 ; Q_{1}}+\left\|u_{m}\right\|_{L_{2}\left(Q_{1}\right)}\right) .
\end{aligned}
$$

Since $D u_{m} \rightarrow D u$ a.e. and $u_{m} \rightarrow u$ in $L_{2}$ in $Q_{1}$, by taking the limit in the above inequality we get (2.3).

Step 2: Continuity of $D_{x^{\prime}} u$ and $\hat{U}$. Next, we prove the second claim of the theorem. Fix a point $z_{0} \in \overline{Q_{1 / 4}}$ and take $0<r<R \leq 1 / 4$.

We define $\omega(R)=\omega_{g, z^{\prime}}(R)+\omega_{B, z^{\prime}}(R)+\omega_{A, z^{\prime}}(R)+R^{\gamma}$, which is a Dini function. By the estimates on $u$ and $D u$ which have already been established, we get from (4.10) that

$$
\begin{aligned}
& f_{Q_{r}\left(z_{0}\right)}\left|D_{x^{\prime}} u-\left(D_{x^{\prime}} u\right)_{Q_{r}\left(z_{0}\right)}\right|^{2}+\left|\hat{U}-(\hat{U})_{Q_{r}\left(z_{0}\right)}\right|^{2} d z \\
& \leq N_{1}(r / R)^{2 \gamma} \int_{Q_{R}\left(z_{0}\right)}\left|D_{x^{\prime}} u-\left(D_{x^{\prime}} u\right)_{Q_{R}\left(z_{0}\right)}\right|^{2}+\left|\hat{U}-(\hat{U})_{Q_{R}\left(z_{0}\right)}\right|^{2} d z \\
& +N_{4} \omega(R)(R / r)^{d+2},
\end{aligned}
$$

where $N_{4}$ is independent of $r, R$ and $z_{0}$. Again we take $r=\tau R$ with $\tau=$ $\tau(d, n, \nu)$ sufficiently small such that $N_{1} \tau^{2 \gamma}<1 / 2$. By an iteration, we 
obtain from (4.17)

$$
\begin{aligned}
& f_{Q_{\tau^{k}}\left(z_{0}\right)}\left|D_{x^{\prime}} u-\left(D_{x^{\prime}} u\right)_{Q_{r}\left(z_{0}\right)}\right|^{2}+\left|\hat{U}-(\hat{U})_{Q_{r}\left(z_{0}\right)}\right|^{2} d z \\
& \leq 2^{-k} f_{Q_{R}\left(z_{0}\right)}\left|D_{x^{\prime}} u-\left(D_{x^{\prime}} u\right)_{Q_{R}\left(z_{0}\right)}\right|^{2}+\left|\hat{U}-(\hat{U})_{Q_{R}\left(z_{0}\right)}\right|^{2} d z \\
& +N_{4} \sum_{j=1}^{k} 2^{-j} \omega\left(\tau^{k-j} R\right) .
\end{aligned}
$$

We set $R=1 / 4$ and use Lemma 3.1 to conclude that for $r \in(0,1 / 4)$

$$
f_{Q_{r}\left(z_{0}\right)}\left|D_{x^{\prime}} u-\left(D_{x^{\prime}} u\right)_{Q_{r}\left(z_{0}\right)}\right|^{2}+\left|\hat{U}-(\hat{U})_{Q_{r}\left(z_{0}\right)}\right|^{2} d z \leq \tilde{\omega}(r)
$$

where $\tilde{\omega}$ is a Dini function. It then follows from (6.3) of 18 that $D_{x^{\prime}} u$ and $\hat{U}$ are uniformly continuous in $\overline{Q_{1 / 4}}$. By using a dilation and covering argument, the continuity of $D_{x^{\prime}} u$ and $\hat{U}$ in $\overline{Q_{1 / 2}}$ follows.

\section{Systems with Partially Hölder coefficients}

We prove Theorem 2.2 in this section. Thanks to Theorem 2.1, we only need to estimate the last three terms on the left-hand side of (2.4). We estimate them separately by using different methods.

5.1. Estimate of $D_{x^{\prime}} u$ and $\hat{U}$. We fix a point $z_{0} \in Q_{1 / 4}$ and take $0<r<$ $R \leq 1 / 4$. Following the proof of Theorem 2.1, we take $\gamma_{1}=\delta$ and $\gamma \in(\delta, 1)$. Note that under the conditions of Theorem 2.2, we have

$$
\omega_{g, z^{\prime}}(R) \leq[g]_{z^{\prime}, \delta / 2, \delta} R^{\delta}
$$

and similar inequalities for $\omega_{A, z^{\prime}}(R)$ and $\omega_{B, z^{\prime}}(R)$. Owing to (4.10), we get

$$
\begin{aligned}
& \int_{Q_{r}\left(z_{0}\right)}\left|D_{x^{\prime}} u-\left(D_{x^{\prime}} u\right)_{Q_{r}\left(z_{0}\right)}\right|^{2}+\left|\hat{U}-(\hat{U})_{Q_{r}\left(z_{0}\right)}\right|^{2} d z \\
& \leq N_{1}(r / R)^{d+2+2 \gamma} \int_{Q_{R}\left(z_{0}\right)}\left|D_{x^{\prime}} u-\left(D_{x^{\prime}} u\right)_{Q_{R}\left(z_{0}\right)}\right|^{2}+\left|\hat{U}-(\hat{U})_{Q_{R}\left(z_{0}\right)}\right|^{2} d z \\
& +N_{2} R^{d+2+2 \delta}\left([g]_{z^{\prime}, \delta / 2, \delta}+|\mathfrak{f}|_{0 ; Q_{R}\left(z_{0}\right)}+|u|_{\delta / 2, \delta ; Q_{3 / 4}}+|D u|_{0 ; Q_{R}\left(z_{0}\right)}\right)^{2}
\end{aligned}
$$

where $N_{1}$ only depends on $d, n$ and $\nu$, and $N_{2}$ also depends on the $C_{z^{\prime}}^{\delta / 2, \delta}$ semi-norms of $A$ and $B$. 
Since (5.1) holds for any $0<r<R \leq 1 / 4$ and $\delta<\gamma$, by a well-known iteration argument (see e.g., [9, Lemma 2.1, p. 86]),

$$
\begin{aligned}
& \int_{Q_{r}\left(z_{0}\right)}\left|D_{x^{\prime}} u-\left(D_{x^{\prime}} u\right)_{Q_{r}\left(z_{0}\right)}\right|^{2}+\left|\hat{U}-(\hat{U})_{Q_{r}\left(z_{0}\right)}\right|^{2} d z \\
& \leq N_{1} r^{d+2+2 \delta} \int_{Q_{1 / 4}\left(z_{0}\right)}\left|D_{x^{\prime}} u-\left(D_{x^{\prime}} u\right)_{Q_{1 / 4}\left(z_{0}\right)}\right|^{2}+\left|\hat{U}-(\hat{U})_{Q_{1 / 4}\left(z_{0}\right)}\right|^{2} d z \\
& +N_{2} r^{d+2+2 \delta}\left([g]_{z^{\prime}, \delta / 2, \delta ; Q_{1}}+|\mathfrak{f}|_{0 ; Q_{R}\left(z_{0}\right)}+|u|_{\delta / 2, \delta ; Q_{3 / 4}}+|D u|_{0 ; Q_{R}\left(z_{0}\right)}\right)^{2}
\end{aligned}
$$

By the definition of $\mathfrak{f}$ and $U_{e}$, we get from (5.2), Theorem 2.1, Corollary 3.5 that

$$
\begin{aligned}
& \int_{Q_{r}\left(z_{0}\right)}\left|D_{x^{\prime}} u-\left(D_{x^{\prime}} u\right)_{Q_{r}\left(z_{0}\right)}\right|^{2}+\left|\hat{U}-(\hat{U})_{Q_{r}\left(z_{0}\right)}\right|^{2} d z \\
& \leq N_{2} r^{d+2+2 \delta}\left(|g|_{z^{\prime}, \delta / 2, \delta ; Q_{1}}+|f|_{0 ; Q_{1}}+\|u\|_{L_{2}\left(Q_{1}\right)}\right)^{2} .
\end{aligned}
$$

Since (5.3) holds for any $r \in(0,1 / 4)$ and $z_{0} \in Q_{1 / 4}$, by Campanato's characterization of Hölder continuous functions, we obtain

$$
\left[D_{x^{\prime}} u\right]_{\delta / 2, \delta ; Q_{1 / 4}}+[\hat{U}]_{\delta / 2, \delta ; Q_{1 / 4}} \leq N_{2}\left(|g|_{z^{\prime}, \delta / 2, \delta ; Q_{1}}+|f|_{0 ; Q_{1}}+\|u\|_{L_{2}\left(Q_{1}\right)}\right) .
$$

This together with a dilation and covering argument gives

$$
\left[D_{x^{\prime}} u\right]_{\delta / 2, \delta ; Q_{1 / 2}}+[\hat{U}]_{\delta / 2, \delta ; Q_{1 / 2}} \leq N_{2}\left(|g|_{z^{\prime}, \delta / 2, \delta ; Q_{1}}+|f|_{0 ; Q_{1}}+\|u\|_{L_{2}\left(Q_{1}\right)}\right) .
$$

5.2. Estimate of $\langle u\rangle_{1+\delta}$. We estimate $\langle u\rangle_{1+\delta}$ by modifying the argument in [5], which in turn is based on an idea by M. Safonov. The argument in [5] uses the maximum principle, which is unavailable for systems. Here we use some estimates established in Section 3 instead.

We denote by $\hat{\mathbb{P}}_{1}$ the set of all functions $p$ on $\mathbb{R}^{d+1}$ of the form

$$
p(z)=p\left(t, x^{1}, x^{d}\right)=\sum_{i=1}^{d-1} \alpha^{i}\left(x^{d}\right) x^{i}+\beta\left(x^{d}\right) .
$$

Then we define the first-order partial Taylor's polynomial with respect to $z^{\prime}=\left(t, x^{\prime}\right)$ of a function $v$ on $\mathbb{R}^{d+1}$ at a point $z_{0}^{\prime}=\left(t_{0}, x_{0}^{\prime}\right)$ as

$$
\hat{T}_{z_{0}^{\prime}}^{1} v\left(z^{\prime}, x^{d}\right):=v\left(z_{0}^{\prime}, x^{d}\right)+\sum_{i=1}^{d-1} D_{i} v\left(z_{0}^{\prime}, x^{d}\right)\left(x^{i}-x_{0}^{i}\right) .
$$

Let

$$
\zeta\left(z^{\prime}\right)=\zeta\left(t, x^{1}, \ldots, x^{d-1}\right)=\eta(t+1) \prod_{i=1}^{d-1} \eta\left(x^{i}\right)
$$


where $\eta$ is a smooth even function on $\mathbb{R}$ with a compact support in $(-1,1)$ satisfying

$$
\int_{\mathbb{R}} \eta(t) d t=1, \quad \int_{\mathbb{R}} t^{2} \eta(t) d t=0
$$

For $\varepsilon>0$ let $\zeta_{\varepsilon}\left(t, x^{\prime}\right)=\varepsilon^{-d-1} \zeta\left(\varepsilon^{-2} t, \varepsilon^{-1} x^{\prime}\right)$ and define a partial mollification of $v$ with respect to $z^{\prime}$ as

$$
\begin{aligned}
\hat{v}^{\varepsilon}\left(t, x^{\prime}, x^{d}\right) & =\int_{\mathbb{R}} \int_{\mathbb{R}^{d-1}} v\left(s, y^{\prime}, x^{d}\right) \zeta_{\varepsilon}\left(t-s, x^{\prime}-y^{\prime}\right) d y^{\prime} d s \\
& =\int_{\mathbb{R}} \int_{\mathbb{R}^{d-1}} v\left(t-\varepsilon^{2} s, x^{\prime}-\varepsilon y^{\prime}, x^{d}\right) \zeta\left(s, y^{\prime}\right) d y^{\prime} d s .
\end{aligned}
$$

By virtue of Taylor's formula, it is not hard to prove the following lemma for partial mollifications (see, e.g., [11, Chapter 3]).

Lemma 5.1. Let $T \in(-\infty, \infty]$ and $\delta \in[0,2]$. Suppose $v \in C_{z^{\prime}}^{\delta / 2, \delta}\left(\mathbb{R}_{T}^{d+1}\right)$. Then for any $\varepsilon>0$,

$$
\begin{gathered}
\varepsilon^{2-\delta} \sup _{\mathbb{R}_{T}^{d+1}}\left|D_{t} \hat{v}^{\varepsilon}\right|+\varepsilon^{1-\delta} \sup _{\mathbb{R}_{T}^{d+1}}\left|D_{x^{\prime}} \hat{v}^{\varepsilon}\right|+\varepsilon^{2-\delta} \sup _{\mathbb{R}_{T}^{d+1}}\left|D_{x^{\prime}}^{2} \hat{v}^{\varepsilon}\right| \leq N[v]_{z^{\prime}, \delta / 2, \delta}, \\
\sup _{\mathbb{R}_{T}^{d+1}}\left|v-\hat{v}^{\varepsilon}\right| \leq N(d, \delta, \eta) \varepsilon^{\delta}[v]_{z^{\prime}, \delta / 2, \delta}
\end{gathered}
$$

where $N=N(d, \delta, \eta)$.

Now we are ready to estimate $\langle u\rangle_{1+\delta}$. First assume that (2.1) holds in $\mathbb{R}_{0}^{d+1}:=\left\{(t, x) \in \mathbb{R}^{d+1}: t<0\right\}$ and $u \in C_{z^{\prime}}^{(1+\delta) / 2,1+\delta}\left(\mathbb{R}_{0}^{d+1}\right)$. Define

$$
\|u\|_{\text {unif }, L_{2, \text { loc }}}:=\sup _{Q_{1}\left(z_{1}\right) \subset \mathbb{R}_{0}^{d+1}}\|u\|_{L_{2}\left(Q_{1}\left(z_{1}\right)\right)} \text {. }
$$

We claim

$$
[u]_{z^{\prime},(1+\delta) / 2,1+\delta} \leq N_{2}\left(|g|_{z^{\prime}, \delta / 2, \delta}+|f|_{0}+\|u\|_{\text {unif }, L_{2, \text { loc }}}\right),
$$

where $N_{2}=N_{2}\left(d, n, \nu, \delta, K,[A]_{z^{\prime}, \delta / 2, \delta},[B]_{z^{\prime}, \delta / 2, \delta}\right)>0$. Take $r>0$. Let $\kappa>4$ be a number to be chosen later. Denote

$$
\mathcal{P}_{0} u=-u_{t}+D_{\alpha}\left(A^{\alpha \beta}\left(0, x^{d}\right) D_{\beta} u\right) .
$$

Then we have

$$
\mathcal{P}_{0} u=\operatorname{div}(\mathfrak{g}+m)+\mathfrak{f},
$$

where

$$
\begin{aligned}
m_{\alpha}^{i}(z) & =\left(A_{i j}^{\alpha \beta}\left(0, x^{d}\right)-A_{i j}^{\alpha \beta}(z)\right) D_{\beta} u^{j}, \\
\mathfrak{g}=\left(\mathfrak{g}_{\alpha}\right), \quad \mathfrak{g}_{\alpha} & :=g_{\alpha}-B^{\alpha} u, \quad \mathfrak{f}:=f-\hat{B}^{\alpha} D_{\alpha} u-C u .
\end{aligned}
$$

Note that

$$
|m| \leq N\left|z^{\prime}\right|^{\delta}[A]_{z^{\prime}, \delta / 2, \delta}|D u|_{0}
$$

Moreover, since the coefficients of $\mathcal{P}_{0}$ are independent of $z^{\prime}$, we have

$$
\mathcal{P}_{0} \hat{u}^{\kappa r}=\operatorname{div}\left(\hat{\mathfrak{g}}^{\kappa r}+\hat{m}^{\kappa r}\right)+\hat{\mathfrak{f}}^{\kappa r} .
$$


Let $v \in \mathcal{H}_{2}^{1}\left(Q_{\kappa r}\right)$ be a weak solution to the equation

$$
\left\{\begin{aligned}
\mathcal{P}_{0} v=\operatorname{div}\left(\mathfrak{g}-\hat{\mathfrak{g}}^{\kappa r}+m-\hat{m}^{\kappa r}\right)+\mathfrak{f}-\hat{\mathfrak{f}}^{\kappa r} & \text { in } Q_{\kappa r} ; \\
v=0 & \text { on } \partial_{p} Q_{\kappa r} .
\end{aligned}\right.
$$

By Lemma 3.2, we get

$$
\|v\|_{L_{2}\left(Q_{\kappa r}\right)} \leq N \kappa r\left\|\mathfrak{g}-\hat{\mathfrak{g}}^{\kappa r}+m-\hat{m}^{\kappa r}\right\|_{L_{2}\left(Q_{\kappa r}\right)}+N(\kappa r)^{2}\left\|\mathfrak{f}-\hat{\mathfrak{f}}^{\kappa r}\right\|_{L_{2}\left(Q_{\kappa r}\right)} .
$$

Corollary 3.5 and a scaling argument yield

$$
\begin{gathered}
|v|_{0 ; Q_{\kappa r / 2}} \leq N \kappa r\left|\mathfrak{g}-\hat{\mathfrak{g}}^{\kappa r}+m-\hat{m}^{\kappa r}\right|_{0 ; Q_{\kappa r}} \\
+N(\kappa r)^{2}\left|\mathfrak{f}-\hat{\mathfrak{f}}^{\kappa r}\right|_{0 ; Q_{\kappa r}}+N(\kappa r)^{-(d+2) / 2}\|v\|_{L_{2}\left(Q_{\kappa r}\right)} .
\end{gathered}
$$

This together with (5.7), (5.6) and Lemma 5.1 yields

$$
|v|_{0 ; Q_{\kappa r / 2}} \leq N(\kappa r)^{1+\delta}\left([\mathfrak{g}]_{z^{\prime}, \delta / 2, \delta}+[A]_{z^{\prime}, \delta / 2, \delta}|D u|_{0}+(\kappa r)^{1-\delta}|\mathfrak{f}|_{0}\right) .
$$

Let $w=u-\hat{u}^{\kappa r}-v$. Then $w$ satisfies $\mathcal{P}_{0} w=0$ in $Q_{\kappa r}$. It follows from Lemma 3.6. Lemma 3.4 and a scaling that

$$
\left|w-\hat{T}_{z_{0}^{\prime}}^{1} w\right|_{0 ; Q_{r}} \leq N r^{2}[w]_{z^{\prime}, 1,2 ; Q_{r}} \leq N \kappa^{-2}|w|_{0 ; Q_{\kappa r / 2}}
$$

With the triangle inequality, (5.8) and Lemma [5.1, we further deduce

$$
\begin{aligned}
\left|w-\hat{T}_{z_{0}^{\prime}}^{1} w\right|_{0 ; Q_{r}} & \leq N \kappa^{-2}\left(|v|_{0 ; Q_{\kappa r / 2}}+\left|u-\hat{u}^{\kappa r}\right|_{0 ; Q_{\kappa r / 2}}\right) \\
& \leq N \kappa^{\delta-1} r^{1+\delta}\left([\mathfrak{g}]_{z^{\prime}, \delta / 2, \delta}+[A]_{z^{\prime}, \delta / 2, \delta}|D u|_{0}+(\kappa r)^{1-\delta}|\mathfrak{f}|_{0}\right) \\
& +N \kappa^{\delta-1} r^{1+\delta}[u]_{z^{\prime},(1+\delta) / 2,1+\delta}
\end{aligned}
$$

By Lemma 5.1, we also get

$$
\begin{aligned}
\left|\hat{u}^{\kappa r}-\hat{T}_{z_{0}^{\prime}}^{1} \hat{u}^{\kappa r}\right|_{0 ; Q_{r}} & \leq N r^{2}\left(\left[D_{x^{\prime}}^{2} \hat{u}^{\kappa r}\right]_{0 ; Q_{r}}+\left[D_{t} \hat{u}^{\kappa r}\right]_{0 ; Q_{r}}\right) \\
& \leq N \kappa^{\delta-1} r^{1+\delta}[u]_{z^{\prime},(1+\delta) / 2,1+\delta}
\end{aligned}
$$

Take $p=\hat{T}_{z_{0}^{\prime}}^{1} w+\hat{T}_{z_{0}^{\prime}}^{1} \hat{u}^{\kappa r} \in \hat{\mathbb{P}}_{1}$. Then from (5.8), (5.9) and (5.10), we get

$$
\begin{aligned}
|u-p|_{0 ; Q_{r}} & \leq|v|_{0 ; Q_{r}}+\left|\hat{u}^{\kappa r}-\hat{T}_{z_{0}^{\prime}}^{1} \hat{u}^{\kappa r}\right|_{0 ; Q_{r}}+\left|w-\hat{T}_{z_{0}^{\prime}}^{1} w\right|_{0 ; Q_{r}} \\
& \leq N(\kappa r)^{1+\delta}\left([\mathfrak{g}]_{z^{\prime}, \delta / 2, \delta}+[A]_{z^{\prime}, \delta / 2, \delta}|D u|_{0}+(\kappa r)^{1-\delta}|\mathfrak{f}|_{0}\right) \\
& +N \kappa^{\delta-1} r^{1+\delta}[u]_{z^{\prime},(1+\delta) / 2,1+\delta}
\end{aligned}
$$

By a shift of the coordinates, we have for any $z_{0} \in \mathbb{R}_{0}^{d+1}$ and $r>0$,

$$
\begin{aligned}
& r^{-1-\delta} \inf _{p \in \hat{\mathbb{P}}_{1}}|u-p|_{0 ; Q_{r}\left(z_{0}\right)} \\
\leq & N \kappa^{1+\delta}\left([\mathfrak{g}]_{z^{\prime}, \delta / 2, \delta}+[A]_{z^{\prime}, \delta / 2, \delta}|D u|_{0}+(\kappa r)^{1-\delta}|\mathfrak{f}|_{0}\right)+N_{1} \kappa^{\delta-1}[u]_{z^{\prime},(1+\delta) / 2,1+\delta}
\end{aligned}
$$


where $N_{1}=N_{1}(d, n, \nu)>0$. We fix a large $\kappa=\kappa(d, n, \nu)$ such that $N_{1} \kappa^{\delta-1}<1 / 2$. For any $r<1 / \kappa$, we get from (5.11)

$$
\begin{aligned}
& r^{-1-\delta} \inf _{p \in \hat{\mathbb{P}}_{1}}|u-p|_{0 ; Q_{r}\left(z_{0}\right)} \\
& \quad \leq N\left([\mathfrak{g}]_{z^{\prime}, \delta / 2, \delta}+[A]_{z^{\prime}, \delta / 2, \delta}|D u|_{0}+|\mathfrak{f}|_{0}\right)+1 / 2[u]_{z^{\prime},(1+\delta) / 2,1+\delta} .
\end{aligned}
$$

By the definition of $\mathfrak{f}$ and $\mathfrak{g}$,

$$
\begin{aligned}
& r^{-1-\delta} \inf _{p \in \hat{\mathbb{P}}_{1}}|u-p|_{0 ; Q_{r}\left(z_{0}\right)} \\
& \leq N\left([g]_{z^{\prime}, \delta / 2, \delta}+|u|_{0}+[u]_{z^{\prime}, \delta / 2, \delta}+|D u|_{0}+|f|_{0}\right)+1 / 2[u]_{z^{\prime},(1+\delta) / 2,1+\delta} \\
& \leq N_{2}\left(|g|_{z^{\prime}, \delta / 2, \delta}+|f|_{0}+\|u\|_{\text {unif }, L_{2, \text { loc }}}\right)+1 / 2[u]_{z^{\prime},(1+\delta) / 2,1+\delta},
\end{aligned}
$$

where $N_{2}=N_{2}\left(d, n, \nu, \delta, K,[A]_{z^{\prime}, \delta / 2, \delta},[B]_{z^{\prime}, \delta / 2, \delta}\right)>0$. In the last inequality, we used Corollary 3.5 and Theorem 2.1. On the other hand, for $r \geq 1 / \kappa$, clearly we have

$$
r^{-1-\delta} \inf _{p \in \hat{\mathbb{P}}_{1}}|u-p|_{0 ; Q_{r}\left(z_{0}\right)} \leq N|u|_{0} .
$$

Thus, (5.12) holds in any case.

Now by first taking the supremum in (5.12) with respect to $r$ and $z_{0}$, and then using the equivalence of parabolic Hölder semi-norms similar to [11, Theorem 8.5.2], we obtain

$$
[u]_{z^{\prime},(1+\delta) / 2,1+\delta} \leq N_{2}\left(|g|_{z^{\prime}, \delta / 2, \delta}+|f|_{0}+\|u\|_{\text {unif }, L_{2, \text { loc }}}\right)+1 / 2[u]_{z^{\prime},(1+\delta) / 2,1+\delta},
$$

which implies (5.5) under the assumption that $u \in C_{z^{\prime}}^{(1+\delta) / 2,1+\delta}\left(\mathbb{R}_{0}^{d+1}\right)$.

Next, we localize the estimate. Let $u$ be a weak solution to (2.1) in $Q_{1}$ and assume $u \in C_{z^{\prime}}^{(1+\delta) / 2,1+\delta}\left(Q_{3 / 4}\right)$. We take a cutoff function $\varsigma$ such that $\varsigma=0$ in $\mathbb{R}_{0}^{d+1} \backslash Q_{3 / 4}$ and $\varsigma=1$ in $Q_{1 / 2}$. Then $\tilde{u}=u \varsigma$ satisfies

$$
\mathcal{P} \tilde{u}=\operatorname{div} \tilde{g}+\tilde{f} \quad \text { in } \mathbb{R}_{0}^{d+1},
$$

where

$$
\begin{aligned}
\tilde{g}_{\alpha} & =g_{\alpha} \varsigma+A^{\alpha \beta} u D_{\beta} \varsigma, \\
\tilde{f} & =f \varsigma-u \varsigma_{t}-g_{\alpha} D_{\alpha} \varsigma+\left(A^{\alpha \beta} D_{\beta} u+\left(B^{\alpha}+\hat{B}^{\alpha}\right) u\right) D_{\alpha} \varsigma .
\end{aligned}
$$

We then have (5.5) with $\tilde{u}, \tilde{f}, \tilde{g}$ in place of $u, f, g$. This together with Theorem 2.1 and Corollary 3.5 yields

$$
[u]_{z^{\prime},(1+\delta) / 2,1+\delta ; Q_{1 / 2}} \leq N_{2}\left(|g|_{z^{\prime}, \delta / 2, \delta ; Q_{1}}+|f|_{0 ; Q_{1}}+\|u\|_{L_{2}\left(Q_{1}\right)}\right) .
$$

Finally, we drop the assumption that $u \in C_{z^{\prime}}^{(1+\delta) / 2,1+\delta}\left(Q_{3 / 4}\right)$ by using the same approximation argument as in the proof of Theorem 2.1. Combining (5.4) and (5.13), the theorem is proved. 


\section{NON-DIVERGENCE FORM EQUATIONS}

In this section, we are concerned with non-divergence scalar parabolic equations

$$
P u:=-u_{t}+a^{\alpha \beta} D_{\alpha \beta} u+b^{\alpha} D_{\alpha} u+c u=f,
$$

The coefficients $a^{\alpha \beta}, b^{\alpha}$, and $c$ are assumed to be measurable and bounded by $K$, and the leading coefficients $a^{\alpha \beta}$ are uniformly elliptic with ellipticity constant $\nu$ :

$$
\nu|\xi|^{2} \leq a^{\alpha \beta} \xi^{\alpha} \xi^{\beta}, \quad\left|a^{\alpha \beta}\right| \leq \nu^{-1}
$$

for any $\xi \in \mathbb{R}^{d}$. The aim here is to prove estimates in the same spirit as in Theorems 2.1 and 2.2 for non-divergence form equations. We state the main results of this section as follows. Theorem 6.2 improves Theorem 2.14 [5] and Theorem 3.1 [19] in the case $q=d-1$.

Theorem 6.1. Let $a, b, c \in C_{z^{\prime}}^{\text {Dini }}$ and $f \in C_{z^{\prime}}^{\text {Dini }}\left(Q_{1}\right)$. Assume that $u \in$ $W_{2}^{1,2}\left(Q_{1}\right)$ is a strong solution to (6.1) in $Q_{1}$. Then we have

$$
|u|_{1,2 ; Q_{1 / 2}} \leq N\left(I\left[\omega_{f, z^{\prime}}\right](1)+|f|_{0 ; Q_{1}}+\|u\|_{L_{2}\left(Q_{1}\right)}\right),
$$

where $N=N\left(d, \nu, K, \omega_{a, z^{\prime}}, \omega_{b, z^{\prime}}, \omega_{c, z^{\prime}}\right)$. Moreover, $u_{t}$ and $D_{x x^{\prime}} u$ are continuous in $\overline{Q_{1 / 2}}$.

Theorem 6.2. Let $\delta \in(0,1), a, b, c \in C_{z^{\prime}}^{\delta / 2, \delta}$ and $f \in C_{z^{\prime}}^{\delta / 2, \delta}\left(Q_{1}\right)$. Assume that $u \in W_{2}^{1,2}\left(Q_{1}\right)$ is a strong solution to (6.1) in $Q_{1}$. Then we have

$$
|u|_{1,2 ; Q_{1 / 2}}+\left[u_{t}\right]_{\delta / 2, \delta ; Q_{1 / 2}}+\left[D_{x x^{\prime}} u\right]_{\delta / 2, \delta ; Q_{1 / 2}} \leq N\left(|f|_{z^{\prime}, \delta / 2, \delta ; Q_{1}}+\|u\|_{L_{2}\left(Q_{1}\right)}\right) \text {, }
$$

where $N=N\left(d, \delta, \nu, K,[a]_{z^{\prime}, \delta / 2, \delta},[b]_{z^{\prime}, \delta / 2, \delta},[c]_{z^{\prime}, \delta / 2, \delta}\right)$.

Remark 6.3. From Theorems 6.1 and 6.2 , we also obtain the corresponding results for non-divergence form elliptic equations, as in Corollaries 2.6] and 2.7.

Remark 6.4. We consider the case when the coefficients and data are piecewise Hölder continuous. Suppose that $Q_{1}$ is divided into $M$ laminate subdomains $\mathcal{D}_{1}, \mathcal{D}_{2}, \cdots, \mathcal{D}_{M}$ by $M-1$ parallel hyperplanes with the common normal direction $(0, \cdots, 0,1)$. Assume $a, b, c$ and $f$ are in $C^{\delta / 2, \delta}\left(\mathcal{D}_{i}\right)$ for each $i=1,2, \cdots, M$, but may have jump discontinuities across these hyperplanes. By Theorem 6.2 and a covering argument, for any $\varepsilon \in(0,1 / 2), u_{t}$ and $D_{x x^{\prime}} u$ are in $C^{\delta / 2, \delta}\left(Q_{1-\varepsilon}\right)$. Moreover, by the same theorem, $u$ and $D u$ are in $C^{\delta / 2, \delta}\left(Q_{1-\varepsilon}\right)$. Now restricted to each $\mathcal{D}_{i} \cap Q_{1-\varepsilon}$, since

$$
D_{d d} u=\left(a^{d d}\right)^{-1}\left(f+u_{t}-b^{\alpha} D_{\alpha} u-c u-\sum_{(\alpha, \beta) \neq(d, d)} a^{\alpha \beta} D_{\alpha \beta} u\right),
$$

we conclude that $D_{d d} u \in C^{\delta / 2, \delta}\left(\mathcal{D}_{i} \cap Q_{1-\varepsilon}\right)$, and hence $u \in C^{1+\delta / 2,2+\delta}\left(\mathcal{D}_{i} \cap\right.$ $\left.Q_{1-\varepsilon}\right)$. It is worth noting that the $C^{1+\delta / 2,2+\delta}$ norm of in each sub-domain $\mathcal{D}_{i} \cap Q_{1-\varepsilon}$ is independent the number $M$. 
The proofs of Theorems 6.1 and 6.2 follow the line of the proofs of Theorems 2.1 and 2.2, and in fact are simpler. First, by using the argument in Section 3, we obtain from the main result of [10] the following interior estimates, which is analogous to Corollary 3.5 .

Lemma 6.5. Let $q \in[2, \infty)$. Assume $a^{\alpha \beta}$ are $V M O$ in $z^{\prime}, u \in C_{l o c}^{\infty}$ satisfies (6.1) in $Q_{1}$, where $f \in L_{q}\left(Q_{1}\right)$. Then there exists a constant $N=N\left(d, \nu, K, \omega_{a, z^{\prime}}, q\right)$ such that

$$
\|u\|_{W_{q}^{1,2}\left(Q_{1 / 2}\right)} \leq N\left(\|u\|_{L_{2}\left(Q_{1}\right)}+\|f\|_{L_{q}\left(Q_{1}\right)}\right) .
$$

In particular, if $q>d+2$, it holds that

$$
|u|_{(1+\gamma) / 2,1+\gamma ; Q_{1 / 2}} \leq N\left(\|u\|_{L_{2}\left(Q_{1}\right)}+\|f\|_{L_{q}\left(Q_{1}\right)}\right),
$$

where $\gamma=1-(d+2) / q$.

We denote

$$
P_{0}(u)=-u_{t}+a^{\alpha \beta} D_{\alpha \beta} u \text {. }
$$

The next lemma is an analogy of Lemma 3.6.

Lemma 6.6. Let $\gamma \in(0,1)$. Assume $u \in C_{\text {loc }}^{\infty}$ satisfies $P_{0} u=f_{0}$ in $Q_{1}$, where $f_{0}$ and $a^{\alpha \beta}$ are independent of $t$ and $x^{\prime}$. Then there exists a constant $N=N(d, \gamma, \nu)$ such that

$$
\begin{aligned}
{\left[u_{t}\right]_{(1+\gamma) / 2,1+\gamma ; Q_{1 / 2}} } & \leq N\left\|u_{t}\right\|_{L_{2}\left(Q_{1}\right)}, \\
{\left[D_{x^{\prime}} u\right]_{(1+\gamma) / 2,1+\gamma ; Q_{1 / 2}} } & \leq N\left\|D_{x x^{\prime}} u\right\|_{L_{2}\left(Q_{1}\right)} .
\end{aligned}
$$

Proof. Note that in $Q_{1}$

$$
P_{0}\left(D_{t}^{i} D_{x^{\prime}}^{j} u\right)=0
$$

wherever $i+j \geq 1$. Then (6.4) follows from Lemma 6.5 applied to $u_{t}$. For any $1 / 2 \leq r<R \leq 1$, applying the same lemma to $D_{x^{\prime}} u-\left(D_{x^{\prime}} u\right)_{Q_{R}}$ gives

$$
\left[D_{x^{\prime}} u\right]_{(1+\gamma) / 2,1+\gamma ; Q_{r}} \leq N\left\|D_{x^{\prime}} u-\left(D_{x^{\prime}} u\right)_{Q_{R}}\right\|_{L_{2}\left(Q_{R}\right)} .
$$

To bound the right-hand side of (6.6), we use an idea in [6] to utilize the divergence structure of the equation after making a change of variables. Let

$$
y^{d}=\varphi\left(x^{d}\right):=\int_{0}^{x^{d}} \frac{1}{a^{d d}(s)} d s, \quad y^{\beta}=x^{\beta}, \beta=1, \cdots, d-1 .
$$

It is easy to see that $\varphi$ is a bi-Lipschitz function and

$$
\delta \leq y^{d} / x^{d} \leq \delta^{-1}, \quad D_{y^{d}}=a^{d d}\left(x^{d}\right) D_{x^{d}} .
$$

Denote

$$
\begin{gathered}
v\left(t, y^{\prime}, y^{d}\right)=u\left(t, y^{\prime}, \varphi^{-1}\left(y^{d}\right)\right), \quad \hat{a}^{i j}\left(y^{d}\right)=a^{i j}\left(\varphi^{-1}\left(y^{d}\right)\right), \\
\hat{f}\left(y^{d}\right)=f\left(\varphi^{-1}\left(y^{d}\right)\right) .
\end{gathered}
$$

Define a divergence form operator $\hat{P}_{0}$ by

$$
\hat{P}_{0} v=-v_{t}+D_{d}\left(\frac{1}{\hat{a}^{d d}} D_{d} v\right)+\sum_{\beta=1}^{d-1} D_{\beta}\left(\frac{\hat{a}^{d \beta}+\hat{a}^{\beta d}}{\hat{a}^{d d}} D_{d} v\right)+\sum_{\alpha, \beta=1}^{d-1} D_{\alpha}\left(\hat{a}^{\alpha \beta} D_{\beta} v\right) .
$$


Clearly, $\tilde{P}_{0}$ is uniformly nondegenerate and $v$ satisfies $\hat{P}_{0} v=\hat{f}$ in some stretched cylindrical domain. Since $\hat{P}_{0}\left(D_{y^{\prime}} v\right)=0$, one can use Lemma 3.3 i) to estimate the mean oscillation of $D_{y^{\prime}} v$ in each parabolic cylinder by the integral of $D_{y y^{\prime}} v$ in the same cylinder. To finish the proof of (6.5), it suffices to use a covering argument bearing in mind that the metrics in $x$-coordinate and $y$-coordinate are comparable.

Now we are ready to prove Theorems 6.1 and 6.2 .

Proof of theorem 6.1. First we assume that $b=c=0$. Also, arguing as before we may assume that $a$ are infinitely differentiable and $u$ has bounded derivative up to fourth order in $Q_{3 / 4}$. We take $0<\gamma<1$. Fix a point $z_{0} \in Q_{3 / 4}$, and take $0<r<R \leq\left(3 / 4-\left|x_{0}\right|\right) / 4$. Now take $z_{1}^{\prime} \in Q_{R}^{\prime}\left(z_{0}^{\prime}\right)$ and denote

Then we have

$$
P_{z_{1}^{\prime}} u=-u_{t}+A^{\alpha \beta}\left(z_{1}^{\prime}, x^{d}\right) D_{\alpha \beta} u \text {. }
$$

$$
P_{z_{1}^{\prime}} u=f+m
$$

where

$$
m(z)=\left(a^{\alpha \beta}\left(z_{1}^{\prime}, x^{d}\right)-a^{\alpha \beta}(z)\right) D_{\alpha \beta} u .
$$

Let $v$ be the strong solution to the equation

$$
\left\{\begin{aligned}
P_{z_{1}^{\prime}} v=f(z)-f\left(z_{1}^{\prime}, x^{d}\right)+m(z) & \text { in } Q_{2 R}\left(z_{0}\right) \\
v=0 & \text { on } \partial_{p} Q_{2 R}\left(z_{0}\right) .
\end{aligned}\right.
$$

We rewrite $P_{z_{1}^{\prime}}$ as a divergence form operator as in Lemma 6.6, then use Lemma 3.2 to get

$$
\|v\|_{L_{2}\left(Q_{2 R}\left(z_{0}\right)\right)} \leq N R^{2}\left\|f-f\left(z_{1}^{\prime}, x^{d}\right)+m\right\|_{L_{2}\left(Q_{2 R}\left(z_{0}\right)\right)} .
$$

This together with Lemma 6.5 gives

$$
\left\|D^{2} v\right\|_{L_{2}\left(Q_{R}\left(z_{0}\right)\right)}+\left\|v_{t}\right\|_{L_{2}\left(Q_{R}\left(z_{0}\right)\right)} \leq N\left\|f-f\left(z_{1}^{\prime}, x^{d}\right)+m\right\|_{L_{2}\left(Q_{2 R}\left(z_{0}\right)\right)} .
$$

Let $w=u-v$. Then $w$ satisfies $P_{z_{1}^{\prime}} w=f\left(z_{1}^{\prime}, x^{d}\right)$ in $Q_{R}\left(z_{0}\right)$. It follows from Lemma 6.6 and a suitable scaling that

$$
\begin{aligned}
& \int_{Q_{r}\left(z_{0}\right)}\left|D_{x x^{\prime}} w-\left(D_{x x^{\prime}} w\right)_{Q_{r}\left(z_{0}\right)}\right|^{2}+\left|w_{t}-\left(w_{t}\right)_{Q_{r}\left(z_{0}\right)}\right|^{2} d z \\
& \leq N(r / R)^{d+2+2 \gamma} \int_{Q_{R}\left(z_{0}\right)}\left|D_{x x^{\prime}} w\right|^{2}+\left|w_{t}\right|^{2} d z .
\end{aligned}
$$

Define

$h\left(x^{d}\right)=\int_{-1}^{x^{d}} \int_{-1}^{s}\left(a^{d d}\left(z_{1}^{\prime}, \tau\right)\right)^{-1}\left(a^{\alpha \beta}\left(z_{1}^{\prime}, \tau\right)\left(D_{\alpha \beta} w\right)_{Q_{R}\left(z_{0}\right)}-\left(w_{t}\right)_{Q_{R}\left(z_{0}\right)}\right) d \tau d s$,

and

$$
\tilde{w}:=w-t\left(w_{t}\right)_{Q_{R}\left(z_{0}\right)}-\frac{1}{2} x^{\alpha} x^{\beta}\left(D_{\alpha \beta} w\right)_{Q_{R}\left(z_{0}\right)}+h\left(x^{d}\right) .
$$

Then, we have

$$
D_{x x^{\prime}} \tilde{w}=D_{x x^{\prime}} w-\left(D_{x x^{\prime}} w\right)_{Q_{R}\left(z_{0}\right)}, \quad \tilde{w}_{t}=w_{t}-\left(w_{t}\right)_{Q_{R}\left(z_{0}\right)} .
$$


It is easily seen that $\tilde{w}$ also satisfies $P_{z_{1}^{\prime}} \tilde{w}=0$ in $Q_{R}\left(z_{0}\right)$. We substitute $w$ in (6.8) by $\tilde{w}$ to get

$$
\begin{aligned}
& \int_{Q_{r}\left(z_{0}\right)}\left|D_{x x^{\prime}} w-\left(D_{x x^{\prime}} w\right)_{Q_{r}\left(z_{0}\right)}\right|^{2}+\left|w_{t}-\left(w_{t}\right)_{Q_{r}\left(z_{0}\right)}\right|^{2} d z \\
& \leq N(r / R)^{d+2+2 \gamma} \int_{Q_{R}\left(z_{0}\right)}\left|D_{x x^{\prime}} w-\left(D_{x x^{\prime}} w\right)_{Q_{R}\left(z_{0}\right)}\right|^{2}+\left|w_{t}-\left(w_{t}\right)_{Q_{R}\left(z_{0}\right)}\right|^{2} d z
\end{aligned}
$$

We combine (6.7) with (6.9) and use the triangle inequality to obtain

$$
\begin{aligned}
& \int_{Q_{r}\left(z_{0}\right)}\left|D_{x x^{\prime}} u-\left(D_{x x^{\prime}} u\right)_{Q_{r}\left(z_{0}\right)}\right|^{2}+\left|D_{t} u-\left(D_{t} u\right)_{Q_{r}\left(z_{0}\right)}\right|^{2} d z \\
& \leq N_{1}(r / R)^{d+2+2 \gamma} \int_{Q_{R}\left(z_{0}\right)}\left|D_{x x^{\prime}} u-\left(D_{x x^{\prime}} u\right)_{Q_{R}\left(z_{0}\right)}\right|^{2}+\left|D_{t} u-\left(D_{t} u\right)_{Q_{r}\left(z_{0}\right)}\right|^{2} d z \\
& +N\left\|f-f\left(z_{1}^{\prime}, x^{d}\right)+m\right\|_{L_{2}\left(Q_{2 R}\left(z_{0}\right)\right)}^{2}
\end{aligned}
$$

where $N_{1}=N_{1}(d, \nu)$. Now we take average of both sides of (6.10) with respect to $z_{1}^{\prime} \in Q_{R}^{\prime}\left(z_{0}^{\prime}\right)$ to get

$$
\begin{aligned}
& \int_{Q_{r}\left(z_{0}\right)}\left|D_{x x^{\prime}} u-\left(D_{x x^{\prime}} u\right)_{Q_{r}\left(z_{0}\right)}\right|^{2}+\left|D_{t} u-\left(D_{t} u\right)_{Q_{r}\left(z_{0}\right)}\right|^{2} d z \\
& \leq N_{1}(r / R)^{d+2+2 \gamma} \int_{Q_{R}\left(z_{0}\right)}\left|D_{x x^{\prime}} u-\left(D_{x x^{\prime}} u\right)_{Q_{R}\left(z_{0}\right)}\right|^{2}+\left|D_{t} u-\left(D_{t} u\right)_{Q_{r}\left(z_{0}\right)}\right|^{2} d z \\
& +N R^{d+2} \omega_{f, z^{\prime}}^{2}(2 R)+N R^{d+2} \omega_{a, z^{\prime}}^{2}(2 R)\left|D^{2} u\right|_{0 ; Q_{2 R}\left(z_{0}\right)}^{2}
\end{aligned}
$$

As in Section 4, we immediately get (6.2) from (6.11) by using an iteration argument and Lemma 6.5. Then the argument in Step 2 of the proof of Theorem 2.1 shows the continuity of $D_{x x^{\prime}} u$ and $u_{t}$.

In the general case, we move all the lower order terms to the right-hand side:

$$
-u_{t}+a^{\alpha \beta} D_{\alpha \beta} u=f-b^{\alpha} D_{\alpha} u-c u:=\mathfrak{f} .
$$

From the proof above, we get

$$
|u|_{1,2 ; Q_{1 / 2}} \leq N\left(I\left[\omega_{\mathfrak{f}, z^{\prime}}\right](1)+|\mathfrak{f}|_{0 ; Q_{1}}+\|u\|_{L_{2}\left(Q_{1}\right)}\right) .
$$

To bound the first two terms on the right-hand side, it suffices to use Lemma 6.5 and the assumptions on $b$ and $c$.

Proof of Theorem 6.2. First we assume that $b=c=0$. We fix a point $z_{0} \in Q_{1 / 4}$ and take $0<r<R \leq 1 / 8$. Following the proof of Theorem 6.1, 
we take $\gamma \in(\delta, 1)$. Owing to (6.10), we get

$$
\begin{aligned}
& \int_{Q_{r}\left(z_{0}\right)}\left|D_{x x^{\prime}} u-\left(D_{x x^{\prime}} u\right)_{Q_{r}\left(z_{0}\right)}\right|^{2}+\left|u_{t}-\left(u_{t}\right)_{Q_{r}\left(z_{0}\right)}\right|^{2} d z \\
& \leq N_{1}(r / R)^{d+2+2 \gamma} \int_{Q_{R}\left(z_{0}\right)}\left|D_{x x^{\prime}} u-\left(D_{x x^{\prime}} u\right)_{Q_{R}\left(z_{0}\right)}\right|^{2}+\left|u_{t}-\left(u_{t}\right)_{Q_{R}\left(z_{0}\right)}\right|^{2} d z \\
& +N_{2}\left([f]_{z^{\prime}, \delta / 2, \delta ; Q_{1}}+\left|D^{2} u\right|_{0 ; Q_{2 R}\left(z_{0}\right)}\right)^{2} R^{d+2+2 \delta}
\end{aligned}
$$

where $N_{1}$ only depends on $d$ and $\nu$, and $N_{2}$ also depends on the $C_{z^{\prime}}^{\delta / 2, \delta}$ seminorm of $a$. Since (6.12) holds for any $0<r<R \leq 1 / 8$ and $\delta<\gamma$, by a well-known iteration argument (see e.g., [9, Lemma 2.1, p. 86]),

$$
\begin{aligned}
& \int_{Q_{r}\left(z_{0}\right)}\left|D_{x x^{\prime}} u-\left(D_{x x^{\prime}} u\right)_{Q_{r}\left(z_{0}\right)}\right|^{2}+\left|u_{t}-\left(u_{t}\right)_{Q_{r}\left(z_{0}\right)}\right|^{2} d z \\
& \leq N_{1} r^{d+2+2 \delta} \int_{Q_{1 / 4}\left(z_{0}\right)}\left|D_{x x^{\prime}} u-\left(D_{x x^{\prime}} u\right)_{Q_{1 / 4}\left(z_{0}\right)}\right|^{2}+\left|u_{t}-\left(u_{t}\right)_{Q_{1 / 4}\left(z_{0}\right)}\right|^{2} d z \\
& +N_{2} r^{d+2+2 \delta}\left([f]_{z^{\prime}, \delta / 2, \delta ; Q_{1}}+\left|D^{2} u\right|_{0 ; Q_{2 R}\left(z_{0}\right)}\right)^{2} .
\end{aligned}
$$

We get from (6.13), Theorem 6.1 and Lemma 6.5 that

$$
\begin{aligned}
& \int_{Q_{r}\left(z_{0}\right)}\left|D_{x x^{\prime}} u-\left(D_{x x^{\prime}} u\right)_{Q_{r}\left(z_{0}\right)}\right|^{2}+\left|u_{t}-\left(u_{t}\right)_{Q_{r}\left(z_{0}\right)}\right|^{2} d z \\
& \leq N_{2} r^{d+2+2 \delta}\left(|f|_{z^{\prime}, \delta / 2, \delta ; Q_{1}}+\|u\|_{L_{2}\left(Q_{1}\right)}\right)^{2} .
\end{aligned}
$$

Since (6.14) holds for any $r \in(0,1 / 8)$ and $z_{0} \in Q_{1 / 4}$, by Campanato's characterization of Hölder continuous functions, we obtain

$$
\left[D_{x x^{\prime}} u\right]_{\delta / 2, \delta ; Q_{1 / 4}}+\left[u_{t}\right]_{\delta / 2, \delta ; Q_{1 / 4}} \leq N_{2}\left(|f|_{z^{\prime}, \delta / 2, \delta ; Q_{1}}+\|u\|_{L_{2}\left(Q_{1}\right)}\right) .
$$

This together with a dilation and covering argument gives

$$
\left[D_{x x^{\prime}} u\right]_{\delta / 2, \delta ; Q_{1 / 2}}+\left[u_{t}\right]_{\delta / 2, \delta ; Q_{1 / 2}} \leq N_{2}\left(|f|_{z^{\prime}, \delta / 2, \delta ; Q_{1}}+\|u\|_{L_{2}\left(Q_{1}\right)}\right) .
$$

In the general case, we move all the lower order terms to the right-hand side and ague as before.

We finish this section by proving the following "partial" Schauder estimates. These results generalize Theorem 5.1 [5] and Theorem 2.1 [19] for the Poisson equation.

Let us introduce a few more notation. Let $q$ be an integer such that $1 \leq$ $q \leq d-1$. We distinguish the first $q$ coordinates of $x$ from the rest and write $x=(\tilde{x}, \check{x})$, where $\tilde{x}=\left(x^{1}, \cdots, x^{q}\right)$ and $\check{x}=\left(x^{q+1}, \cdots, x^{d}\right)$. We also denote $\tilde{z}=(t, \tilde{x})$. As in Section 2, we introduce the partial Dini continuous space $C_{\tilde{z}}^{\text {Dini }}(\mathcal{D})$ and the partial Hölder space $C_{\tilde{z}}^{\delta / 2, \delta}$, as well as their corresponding norms. 
Theorem 6.7. Let $a=a\left(x^{d}\right)$ be a measurable function of $x^{d}$ alone. Let $b, c \in C_{\tilde{z}}^{\text {Dini }}$ and $f \in C_{\tilde{z}}^{\text {Dini }}\left(Q_{1}\right)$. Assume that $u \in W_{2}^{1,2}\left(Q_{1}\right)$ is a strong solution to (6.1) in $Q_{1}$. Then we have

$$
\left|u_{t}\right|_{0 ; Q_{1 / 2}}+\left|D_{x \tilde{x}} u\right|_{0 ; Q_{1 / 2}} \leq N\left(I\left[\omega_{f, z^{\prime}}\right](1)+|f|_{0 ; Q_{1}}+\|u\|_{L_{2}\left(Q_{1}\right)}\right),
$$

where $N=N\left(d, \nu, K, \omega_{b, \tilde{z}}, \omega_{c, \tilde{z}}\right)$. Moreover, $u_{t}$ and $D_{x \tilde{x}} u$ are continuous in $\overline{Q_{1 / 2}}$.

Theorem 6.8. Let $\delta \in(0,1), a=a\left(x^{d}\right)$ be a measurable function of $x^{d}$ alone. Let $b, c \in C_{\tilde{z}}^{\delta / 2, \delta}$ and $f \in C_{\tilde{z}}^{\delta / 2, \delta}\left(Q_{1}\right)$. Assume that $u \in W_{2}^{1,2}\left(Q_{1}\right)$ is a strong solution to (6.1) in $Q_{1}$. Then we have

$$
\left|u_{t}\right|_{\delta / 2, \delta ; Q_{1 / 2}}+\left|D_{x \tilde{x}} u\right|_{\delta / 2, \delta ; Q_{1 / 2}} \leq N\left(|f|_{\tilde{z}, \delta / 2, \delta ; Q_{1}}+\|u\|_{L_{2}\left(Q_{1}\right)}\right),
$$

where $N=N\left(d, \delta, \nu, K,[b]_{\tilde{z}, \delta / 2, \delta},[c]_{\tilde{z}, \delta / 2, \delta}\right)$.

For the proofs of Theorems 6.7 and 6.8, first we note that similar to Lemma 6.6 if $f_{0}$ is independent of $\tilde{z}$, then (6.4) still holds and we have

$$
\left[D_{\tilde{x}} u\right]_{(1+\gamma) / 2,1+\gamma ; Q_{1 / 2}} \leq N\left\|D_{x \tilde{x}} u\right\|_{L_{2}\left(Q_{1}\right)} .
$$

Following the proof of Theorem 6.1, first we assume there is no lower order terms. Let $v$ be the solution of

$$
\left\{\begin{aligned}
P_{0} v=f(z)-f\left(\tilde{z}_{1}, \check{x}\right) & \text { in } Q_{2 R}\left(z_{0}\right) ; \\
v=0 & \text { on } \partial_{p} Q_{2 R}\left(z_{0}\right),
\end{aligned}\right.
$$

where $P_{0}$ is defined in (6.3). Then as before we have

$$
\left\|D^{2} v\right\|_{L_{2}\left(Q_{R}\left(z_{0}\right)\right)}+\left\|v_{t}\right\|_{L_{2}\left(Q_{R}\left(z_{0}\right)\right)} \leq N\left\|f-f\left(\tilde{z}_{1}, \check{x}\right)\right\|_{L_{2}\left(Q_{2 R}\left(z_{0}\right)\right)} .
$$

Moreover, $w=u-v$ satisfies

$$
\begin{aligned}
& \int_{Q_{r}\left(z_{0}\right)}\left|D_{x \tilde{x}} w-\left(D_{x \tilde{x}} w\right)_{Q_{r}\left(z_{0}\right)}\right|^{2}+\left|w_{t}-\left(w_{t}\right)_{Q_{r}\left(z_{0}\right)}\right|^{2} d z \\
& \leq N(r / R)^{d+2+2 \gamma} \int_{Q_{R}\left(z_{0}\right)}\left|D_{x \tilde{x}} w-\left(D_{x \tilde{x}} w\right)_{Q_{R}\left(z_{0}\right)}\right|^{2}+\left|w_{t}-\left(w_{t}\right)_{Q_{R}\left(z_{0}\right)}\right|^{2} d z .
\end{aligned}
$$

Combining (6.15) with (6.16) and taking average in $\tilde{z}_{1}$ gives

$$
\begin{aligned}
& \int_{Q_{r}\left(z_{0}\right)}\left|D_{x \tilde{x}} u-\left(D_{x \tilde{x}} u\right)_{Q_{r}\left(z_{0}\right)}\right|^{2}+\left|D_{t} u-\left(D_{t} u\right)_{Q_{r}\left(z_{0}\right)}\right|^{2} d z \\
& \leq N_{1}(r / R)^{d+2+2 \gamma} \int_{Q_{R}\left(z_{0}\right)}\left|D_{x \tilde{x}} u-\left(D_{x \tilde{x}} u\right)_{Q_{R}\left(z_{0}\right)}\right|^{2}+\left|D_{t} u-\left(D_{t} u\right)_{Q_{r}\left(z_{0}\right)}\right|^{2} d z \\
& +N \omega_{f, \tilde{z}}^{2}(2 R) R^{d+2} .
\end{aligned}
$$

Now a standard iteration argument finishes the proof.

We remark in Theorems 6.7 and 6.8 if $b, c$ and $f$ are assumed to be regular only with respect to $\tilde{x}$, we can still get the estimate of $D_{x \tilde{x}} u$ by dropping the $u_{t}$ terms in (6.15) and (6.16) and replacing $f\left(\tilde{z}_{1}, \check{x}\right)$ by $f\left(t, \tilde{x}_{1}, \check{x}\right)$. 


\section{Appendix}

In the appendix, we give a proof of Lemma 3.4. Let $\lambda_{0}$ be the constant in Theorem 2.2 of 4 . Let

$$
r_{k}=1-2^{-k}, \quad Q_{k}=\left(-r_{k}^{2}, 0\right) \times B_{r_{k}}, \quad k=1,2, \cdots .
$$

Then we find $\zeta_{k}(t, x) \in C_{0}^{\infty}\left(\mathbb{R}^{d+1}\right)$ such that

$$
\zeta_{k}=\left\{\begin{array}{lll}
1 & \text { on } & Q_{k} \\
0 & \text { on } & \mathbb{R}^{d+1} \backslash\left(-r_{k+1}^{2}, r_{k+1}^{2}\right) \times B_{r_{k+1}}
\end{array}\right.
$$

and

$$
\left|D \zeta_{k}\right| \leq N 2^{k}, \quad\left|\left(\zeta_{k}\right)_{t}\right| \leq N 2^{2 k}, \quad\left|D^{2} \zeta_{k}\right| \leq N 2^{2 k}
$$

Observe that, for $\lambda_{k} \geq \lambda_{0}$,

$$
\left(\mathcal{P}_{0}-\lambda_{k}\right)\left(\zeta_{k} u\right)=\operatorname{div} g_{k}+f_{k} \quad \text { in } \quad \mathbb{R}_{0}^{d+1},
$$

where

$$
\begin{gathered}
g_{k}=\left(g_{k_{\alpha}}\right), \quad g_{k_{\alpha}}=\zeta_{k} g_{\alpha}+\sum_{\beta=1}^{d} A^{\alpha \beta} u D_{\beta} \zeta_{k}, \\
f_{k}=\zeta_{k} f-\sum_{\alpha=1}^{d} g_{\alpha} D_{\alpha} \zeta_{k}+\sum_{\alpha, \beta=1}^{d} A^{\alpha \beta} D_{\beta} u D_{\alpha} \zeta_{k}-u D_{t} \zeta_{k}-\lambda_{k} u \zeta_{k} .
\end{gathered}
$$

Then by Theorem 2.2 of [4],

$$
\begin{aligned}
& \left\|D\left(\zeta_{k} u\right)\right\|_{L_{p}\left(\mathbb{R}_{0}^{d+1}\right)} \leq N\left(\left\|g_{k}\right\|_{L_{p}\left(\mathbb{R}_{0}^{d+1}\right)}+\lambda_{k}^{-1 / 2}\left\|f_{k}\right\|_{L_{p}\left(\mathbb{R}_{0}^{d+1}\right)}\right) \\
& \leq N\left(2^{k}+\lambda_{k}^{-1 / 2} 2^{2 k}+\lambda_{k}^{1 / 2}\right)\|u\|_{L_{p}\left(Q_{1}\right)}+N \lambda_{k}^{-1 / 2}\|f\|_{L_{p}\left(Q_{1}\right)} \\
& \quad+N\left(1+\lambda_{k}^{-1 / 2} 2^{k}\right)\|g\|_{L_{p}\left(Q_{1}\right)}+N \lambda_{k}^{-1 / 2} 2^{k}\left\|D\left(\zeta_{k+1} u\right)\right\|_{L_{p}\left(\mathbb{R}_{0}^{d+1}\right)} .
\end{aligned}
$$

Set

$$
\mathcal{A}_{k}=\left\|D\left(\zeta_{k} u\right)\right\|_{L_{p}\left(\mathbb{R}_{0}^{d+1}\right)}, \mathcal{B}=\|u\|_{L_{p}\left(Q_{1}\right)}, \mathcal{G}=\|g\|_{L_{p}\left(Q_{1}\right)}, \mathcal{F}=\|f\|_{L_{p}\left(Q_{1}\right)} .
$$

Then

$$
\begin{aligned}
\mathcal{A}_{k} \leq & N\left(2^{k}+\lambda_{k}^{-1 / 2} 2^{2 k}+\lambda_{k}^{1 / 2}\right) \mathcal{B} \\
& +N\left(1+\lambda_{k}^{-1 / 2} 2^{k}\right) \mathcal{G}+N \lambda_{k}^{-1 / 2} \mathcal{F}+N \lambda_{k}^{-1 / 2} 2^{k} \mathcal{A}_{k+1} .
\end{aligned}
$$

By multiplying $\varepsilon^{k}$ both sides and summing up with respect to $k$, we have

$$
\begin{aligned}
& \sum_{k=1}^{\infty} \varepsilon^{k} \mathcal{A}_{k} \leq N \mathcal{B} \sum_{k=1}^{\infty}\left(2^{k}+\lambda_{k}^{-1 / 2} 2^{2 k}+\lambda_{k}^{1 / 2}\right) \varepsilon^{k} \\
& +N \mathcal{G} \sum_{k=1}^{\infty}\left(1+\lambda_{k}^{-1 / 2} 2^{k}\right) \varepsilon^{k}+N \mathcal{F} \sum_{k=1}^{\infty} \lambda_{k}^{-1 / 2} \varepsilon^{k}+N_{1} \sum_{k=1}^{\infty} \lambda_{k}^{-1 / 2}(2 \varepsilon)^{k} \mathcal{A}_{k+1} .
\end{aligned}
$$


We may certainly assume $N_{1} \geq \lambda_{0}^{1 / 2}$. Now set

$$
\varepsilon=1 / 4, \quad \lambda_{k}^{1 / 2}=N_{1} 2^{k+2} .
$$

Then

$$
\begin{aligned}
& \sum_{k=1}^{\infty}\left(2^{k}+\lambda_{k}^{-1 / 2} 2^{2 k}+\lambda_{k}^{1 / 2}\right) \varepsilon^{k} \leq N, \quad \sum_{k=1}^{\infty}\left(1+\lambda_{k}^{-1 / 2} 2^{k}\right) \varepsilon^{k} \leq N \\
& \sum_{k=1}^{\infty} \lambda_{k}^{-1 / 2} \varepsilon^{k} \leq N, \quad N_{1} \sum_{k=1}^{\infty} \lambda_{k}^{-1 / 2}(2 \varepsilon)^{k} \mathcal{A}_{k+1}=\sum_{k=1}^{\infty} \varepsilon^{k+1} \mathcal{A}_{k+1}=\sum_{k=2}^{\infty} \varepsilon^{k} \mathcal{A}_{k} .
\end{aligned}
$$

Therefore,

$$
\sum_{k=1}^{\infty} \varepsilon^{k} \mathcal{A}_{k} \leq N(\mathcal{B}+\mathcal{G}+\mathcal{F})+\sum_{k=2}^{\infty} \varepsilon^{k} \mathcal{A}_{k} .
$$

On the other hand the summations above are finite because

$$
\mathcal{A}_{k} \leq N 2^{k}\|u\|_{L_{p}\left(Q_{1}\right)}+N\|D u\|_{L_{p}\left(Q_{1}\right)} .
$$

Then the inequality (7.1) implies that

$\|D u\|_{L_{p}\left(Q_{1 / 2}\right)} \leq \mathcal{A}_{1} \leq N(\mathcal{B}+\mathcal{G}+\mathcal{F})=N\left(\|u\|_{L_{p}\left(Q_{1}\right)}+\|g\|_{L_{p}\left(Q_{1}\right)}+\|f\|_{L_{p}\left(Q_{1}\right)}\right)$.

Finally, the estimate of $\left\|u_{t}\right\|_{\mathbb{H}_{p}^{-1}\left(Q_{1 / 2}\right)}$ follows from the above estimate and the equation (2.1) itself. The lemma is proved.

\section{ACKNOWLEDGEMENT}

The author is grateful to Nicolai V. Krylov, Yanyan Li, and Mikhail V. Safonov for their interests in this work and helpful comments. He would also like to thank the referee for a very careful reading of the manuscript and many useful comments.

\section{REFERENCES}

[1] E. Bonnetier, M. Vogelius, An elliptic regurality result for a composite medium with touching fibers of circular cross-section, SIAM J. Math. Anal. 31 (2000), 651-677.

[2] I. Babuška, B. Andersson, P. Smith, K. Levin, Damage analysis of fiber composites. I. Statistical analysis on fiber scale, Comput. Methods Appl. Mech. Engrg. 172 (1999), no. $1-4,27-77$.

[3] M. Chipot, D. Kinderlehrer, G. Vergara-Caffarelli, Smoothness of linear laminates, Arch. Rational Mech. Anal. 96 (1986), no. 1, 81-96.

[4] H. Dong, D. Kim, Parabolic and elliptic systems in divergence form with variably partially BMO coefficients, SIAM J. Math. Anal., 43 (2011) no. 3, 1075-1098.

[5] H. Dong, S. Kim, Partial Schauder estimates for second-order elliptic and parabolic equations, Calc. Var. Partial Differential Equations, 40 (2011) no. 3-4, 481-500.

[6] H. Dong, Solvability of second-order equations with hierarchically partially BMO coefficients, Trans. Amer. Math. Soc., 364 (2012) no. 1, 493-517.

[7] J. Fan, K. Kim, M. Nagayasu, G. Nakamura, A gradient estimate for solutions to parabolic equations with discontinuous coefficients, preprint, arXiv:1103.0832

[8] P. Fife, Schauder estimates under incomplete Hölder continuity assumptions, Pacific J. Math. 13 (1963) 511-550. 
[9] M. Giaquinta, Multiple integrals in the calculus of variations and nonlinear elliptic systems. Princeton University Press:Princeton, NJ, 1983.

[10] D. Kim, N. V. Krylov, Parabolic equations with measurable coefficients, Potential Anal. 26 (2007), no. 4, 345-361.

[11] N. V. Krylov, Lectures on elliptic and parabolic equations in Hölder spaces. American Mathematical Society, Providence, RI, 1996.

[12] N. V. Krylov, Parabolic and elliptic equations with VMO coefficients, Comm. Partial Differential Equations 32 (2007), no. 3, 453-475.

[13] Y.Y. Li, M. Vogelius, Gradient estimates for solutions to divergence form elliptic equations with discontinuous coefficients, Arch. Rational Mech. Anal. 153 (2000), no. $2,91-151$.

[14] Y.Y. Li, L. Nirenberg, Estimates for elliptic systems from composite material, Comm. Pure Appl. Math. 56 (2003), no. 7, 892-925.

[15] Y.Y. Li, H. Li, Gradient estimates for parabolic systems from composite material, preprint.

[16] G. M. Lieberman, Intermediate Schauder theory for second order parabolic equations. IV. Time irregularity and regularity, Differential Integral Equations 5 (1992), no. 6, $1219-1236$.

[17] G. M. Lieberman, Second order parabolic differential equations, World Scientific Publishing Co., Inc., River Edge, NJ, 1996.

[18] E. Sperner, Schauder's existence theorem for $\alpha$-Dini continuous data, Ark. Mat. 19 (1981), no. 2, 193-216.

[19] G. Tian, X.-J. Wang, Partial regularity for elliptic equations, Discret. Contin. Dyn. Syst. 28 (2010), no.3, 899-913.

(H. Dong) Division of Applied Mathematics, Brown University, 182 George Street, Providence, RI 02912, USA

E-mail address: Hongjie_Dong@brown.edu 